\title{
A PARSIMONIOUS BEHAVIORAL SEIR MODEL OF THE 2020 COVID EPIDEMIC IN THE UNITED STATES AND THE UNITED KINGDOM
}

\author{
Andrew Atkeson \\ Working Paper 28434 \\ http://www.nber.org/papers/w28434 \\ NATIONAL BUREAU OF ECONOMIC RESEARCH \\ 1050 Massachusetts Avenue \\ Cambridge, MA 02138 \\ February 2021
}

I acknowledge many useful discussions on this topic for many months. I am grateful in particular to Karen Kopecky, Tao Zha, Jim Stock, and Mike Droste. The views expressed herein are those of the author and do not necessarily reflect the views of the National Bureau of Economic Research.

NBER working papers are circulated for discussion and comment purposes. They have not been peer-reviewed or been subject to the review by the NBER Board of Directors that accompanies official NBER publications.

(C) 2021 by Andrew Atkeson. All rights reserved. Short sections of text, not to exceed two paragraphs, may be quoted without explicit permission provided that full credit, including () notice, is given to the source. 
A Parsimonious Behavioral SEIR Model of the 2020 COVID Epidemic in the United States and the United Kingdom

Andrew Atkeson

NBER Working Paper No. 28434

February 2021

JEL No. C0,I0

\begin{abstract}
I present a behavioral epidemiological model of the evolution of the COVID epidemic in the United States and the United Kingdom over the past 12 months. The model includes the introduction of a new, more contagious variant in the UK in early fall and the US in mid December. The model is behavioral in that activity, and thus transmission, responds endogenously to the daily death rate. I show that with only seasonal variation in the transmission rate and pandemic fatigue modeled as a one-time reduction in the semi-elasticity of the transmission rate to the daily death rate late in the year, the model can reproduce the evolution of daily and cumulative COVID deaths in the both countries from Feb 15, 2020 to the present remarkably well. I find that most of the end-of-year surge in deaths in both the US and the UK was generated by pandemic fatigue and not the new variant of the virus. I then generate forecasts for the evolution of the epidemic over the next two years with continuing seasonality, pandemic fatigue, and spread of the new variant.
\end{abstract}

Andrew Atkeson

Bunche Hall 9381

Department of Economics

UCLA

Box 951477

Los Angeles, CA 90095-1477

and NBER

andy@atkeson.net

A data appendix is available at http://www.nber.org/data-appendix/w28434 


\section{Introduction}

Economists have made a convincing case that epidemiological models should include consideration of the endogenous response of individuals and policymakers to the evolution of the epidemic itself in accounting for the evolution of observed epidemics and in constructing counterfactual outcomes under different policy and disease scenarios. But, to date, economists have not constructed parsimonious models of the interaction of behavior and disease that can account for the observed evolution of the COVID epidemic without introducing large and frequent shocks to the parameters of these models. ${ }^{1}$

I provide such a model in this paper. This model is a an SEIR model (with compartments for agents who are susceptible, S, exposed, E, infectious, I, and recovered R) modified to include a compartment for those infected agents who end up with serious disease. I refer to this compartment as $\mathrm{H}$, for hospitalized. Agents who die from COVID are assumed to transition from infection I to death, D, through this compartment $\mathrm{H}$. The expected time that agents spend in this compartment is set to 30 days to capture the delay between serious illness, death, and the reporting of that death. Behavior in this model is assumed to respond to daily death rates. It is assumed that behavior does not respond immediately to new infections as these are not directly observed. As discussed by John Cochrane ${ }^{2}$ and Weitz et. al. $2020^{3}$ the delay between infection and death introduced by this compartment $\mathrm{H}$ implies that this simple behavioral model has oscillatory endogenous dynamics that are helpful in allowing the model to reproduce the data with only a few shocks.

The two shocks considered in this paper are as follows. First, I add a standard

\footnotetext{
${ }^{1}$ See Atkeson, Kopecky, and Zha 2021 and Droste and Stock 2021 for a discussion the difficulties in doing so. Both of these papers are forthcoming in the AER Papers and Proceedings. The first paper is available here https://www.aeaweb.org/conference/2021/preliminary/paper/SYHZZkBA.

${ }^{2}$ See https://johnhcochrane.blogspot.com/2020/05/an-sir-model-with-behavior.html

${ }^{3}$ Joshua Weitz, Sang Woo Park, Ceyhun Eksin, and Jonathan Dushoff, "Awareness-driven behavior changes can shift the shape of epidemics away from peaks and toward plateaus, shoulders, and oscillations" , Proceedings of the National Academy of Science, vol. 117, no. 51, December 22, 2020
} 
seasonal variation in the baseline transmission rate of the virus from a winter peak to a low in midsummer. Second, I introduce a one-time change in behavior modeled as a reduction in the semi-elasticity of the transmission rate with respect to the daily death rate from an initial level to a new, permanently lower level. I refer to this second shock as the onset of pandemic fatigue. In both countries, pandemic fatigue sets in late in the year.

Finally, for the United Kingdom, I introduce in the model a new, more contagious, variant of COVID that gradually takes over from the initial variant. This variant is introduced into the model specification for the United Kingdom on September 1, 2020. I discipline the model parameters describing the transmissibility of this new variant by comparing the model prediction for the growth of the prevalence of this new variant to data from the UK on the weekly prevalence of this new variant in new COVID tests. ${ }^{4}$ I find that this new variant is $38 \%$ more transmissible than the original variant in the UK holding the impact of seasonality and behavior fixed. I introduce this variant into the United States in late December for purposes of forecasting.

To evaluate the role of each of these two shocks in accounting for the data, I simulate the model with neither shock, with each shock alone, and then with the two shocks combined. I find that seasonality is key in accounting for the relatively low level of daily deaths observed during the summer of 2020 in both countries, and in both countries, the model with a fixed behavioral response and seasonality alone does a remarkable job in matching the pattern of daily deaths through the late summer of 2020. I find that pandemic fatigue is key in accounting for the onset of a large second wave of deaths experienced this Fall and Winter in both countries. In particular, seasonality in transmission is not sufficient to generate a large second wave of deaths because behavior endogenously offsets a gradual rise in the inherent transmission rate of the virus. For the United Kingdom, I find that the specification

\footnotetext{
${ }^{4}$ See this document from the European Center for Disease Prevention and Control on this new UK variant of COVID and Figure 2 in this document for data on its increasing prevalence in the UK over time https://www.ecdc.europa.eu/sites/default/files/documents/ COVID-19-risk-related-to-spread-of-new-SARS-CoV-2-variants-EU-EEA-first-update.pdf.
} 
of the model with seasonality and a new variant but without pandemic fatigue also does not generate the large wave of deaths experienced in that country late in the year. Thus, I conclude that most of that wave was accounted for by pandemic fatigue and not the new variant.

One concern about the results regarding pandemic fatigue found here is that no dramatic change in cell phone mobility data can be seen in those data in the window of time for which my assumed pandemic fatigue shock occurs. This discrepancy between model and data may reflect either a problem with the model or a problem with the cell phone mobility data as an indicator of the impact of human behavior on virus transmission. It is intriguing that the model assumed onset of pandemic fatigue in the United States coincides with the announcement of success of the Phase 3 trials of the Pfizer vaccine in the United States on November 9 and shortly after the announcement of the success of the Phase 3 trials of the Oxford-AstraZeneca vaccine in the United Kingdom on November 23. Perhaps the news of vaccines led to subtle changes in individual behavior that reduced the endogenous responsiveness of the transmission rate to daily deaths.

I also simulate the model with a much shorter expected time in the $\mathrm{H}$ compartment and show that this alternative specification of the model has the same difficulties generating equilibrium variation in the growth rate of daily deaths after the initial phase of the epidemic as found in Atkeson, Kopecky, and Zha 2021. This model experiment confirms that it is critical to include a delayed response of behavior to new infections to have the model match the data.

I then generate a forecast from this model for the evolution of deaths from the epidemic over the course of the next two years by simulating the model from its initial conditions for three full years. In this simulation, I assume that seasonality and pandemic fatigue continue through the second and third years. Specifically, I assume that the semi-elasticity of transmission with respect to daily deaths remains at its new lower level consistent with the second wave of deaths this Fall and Winter. I also introduce the new variant into the model specification for the United States 
on December 1, 2020 for forecasting purposes.

In these forecasts, I do not account for the efficacy of vaccines in reducing either transmission or death (or both). I find that without a successful vaccine program, a substantial third wave of deaths is likely in both countries in the Fall and Winter of 2021 and cumulative deaths would asymptote to a level just shy of 1.25 million in the US with $75 \%$ percent of the population experiencing the virus by late summer 2022 . The analogous forecasts for the United Kingdom are just over 240,000 cumulative deaths and just over $70 \%$ of the population experiencing the virus by late summer 2022. These results indicate that a successfully executed vaccination program could potentially save a large number of lives in both countries, roughly half a million in the United States and one hundred thousand or more in the United Kingdom.

The remainder of this paper is organized as follows. In section 2 , I briefly describe the model and I present the main results. The model is described in detail in section 6 and the MATLAB code to implement the model is included with this paper as an Appendix. ${ }^{5}$ The forecasts generated from the model are presented in section 4 . I briefly discuss these results in section 5 .

\section{Methodology and Main Results}

The model is a standard compartmental SEIR model augmented with the compartment $\mathrm{H}$ for those with serious illness. Behavior is modeled in a reduced-form manner: the transmission rate of the disease is assumed to decline with increasing daily deaths. ${ }^{6}$ The epidemiological parameters of the model are standard and are reported with the model description given after presenting the main results. The choice of a flow rate from hospitalization to death corresponding to an average stay

\footnotetext{
${ }^{5}$ The MATLAB code and data files for this paper are also available at https://sites.google.com/ site/andyatkeson/

${ }^{6}$ Given the structure of the model, this assumption regarding behavior is mathematically equivalent to an assumption that the transmission rate of the disease declines with increasing numbers hospitalized with severe disease.
} 
in the $\mathrm{H}$ compartment of 30 days corresponds to estimates of the mean time from symptom onset to death and from death to reporting of the death suggested by the CDC for pandemic planning scenarios. ${ }^{7}$

In all scenarios, I simulate the model from an initial condition on February 15, 2020 with a small initial number of exposed individuals ${ }^{8}$ and no infected individuals. I simulate the model for an entire year from this initial condition with combinations of the three driving forces described above: seasonality in transmission, pandemic fatigue, and, in the UK, a new variant introduced as 1 exposed individual on September 1, 2020. The new variant is assumed to be $38 \%$ more transmissible than the original variant in the UK, holding fixed the date and behavior. All other epidemiological parameters associated with this new variant are assumed to be the same as for the original variant. In introduce one case of this new variant into the United States on December 1, 2020.

I first compute a baseline simulation with both seasonality in transmission and pandemic fatigue. Results are presented in section 2.1. I also show the assumed patterns of seasonality and pandemic fatigue and I present results for the prevalence over time of the new variant of the virus in the UK.

To assess the role of these shocks and the long delay in the compartment $\mathrm{H}$ in accounting for the data, I then simulate the model three more times.

In the first alternative simulation, in section 2.2, I simulate the model with neither the seasonality nor pandemic fatigue. In this case, we see that the model does a poor job of fitting the data, so it is clear that the two shocks together play a key role in shaping the path of the epidemic. But we also see clearly the endogenous oscillatory dynamics of the model that end up playing a key role in having the model match the data.

\footnotetext{
${ }^{7}$ See https://www.cdc.gov/coronavirus/2019-ncov/hcp/planning-scenarios.html. On that webpage, the CDC notes a median time in the hospital of 2 weeks for those who are admitted to the ICU and a median time of 21 days between death and reporting of the death.

${ }^{8}$ For the US, I use an initial exposure of 33 out of a population of 330 million and in the UK, I use initial exposure of 7 out of a population of 67.9 million
} 
In the second alternative simulation, in section 2.3, I simulate the model with seasonality but no pandemic fatigue. In this case, we see that the model does a good job of matching the data through the summer of 2020, but then fails to match the large second wave of deaths in both countries that occurred late in the year. This observation is striking given that, due to seasonality, the basic reproduction number for the virus rises from 2.1 to 3 in the United States from mid summer to February 1, 2021. This substantial but gradual increase in the underlying transmissibility of the virus does not produce a large wave of deaths because the behavioral response has time to offset it even with the substantial delay between infection and reported death assumed in the model.

The failure of the model to produce a substantial second wave in the United Kingdom is even more striking in that both seasonality and the introduction of the new variant dramatically boost overall transmissibility of the virus late in the year. Again, it is the endogenous response of behavior offsets the increased transmissibility of the virus and thus limits the size of this wave of deaths predicted by the model.

It is based on this finding that I conclude that the new variant in the United Kingdom did not, in and of itself, drive the large wave of deaths that occurred late in the year. It was only with the combination of pandemic fatigue and the new variant that the model matches the size of that wave

In the third alternative simulation, in section 3, I simulate the model with both seasonality and pandemic fatigue, but I dramatically shorten the average time that agents spend in compartment $\mathrm{H}$. Note that this alteration of the model does not directly impact the model-implied dynamics of infection holding behavior fixed. What we see in this simulation is the impact on the evolution of the epidemic of the endogenous change in the dynamics of infection through the delay in behavior induced by the compartment $\mathrm{H}$. 


\subsection{Baseline: with seasonality and pandemic fatigue}

The results of this simulation with both seasonality and pandemic fatigue for daily deaths and cumulative deaths are shown (in blue) together with data (in red) for the United States in Figures 1 and 2. Results for the United Kingdom are presented in Figures 1 and 2. As we can see, the model simulation fits the data remarkably well in both countries.

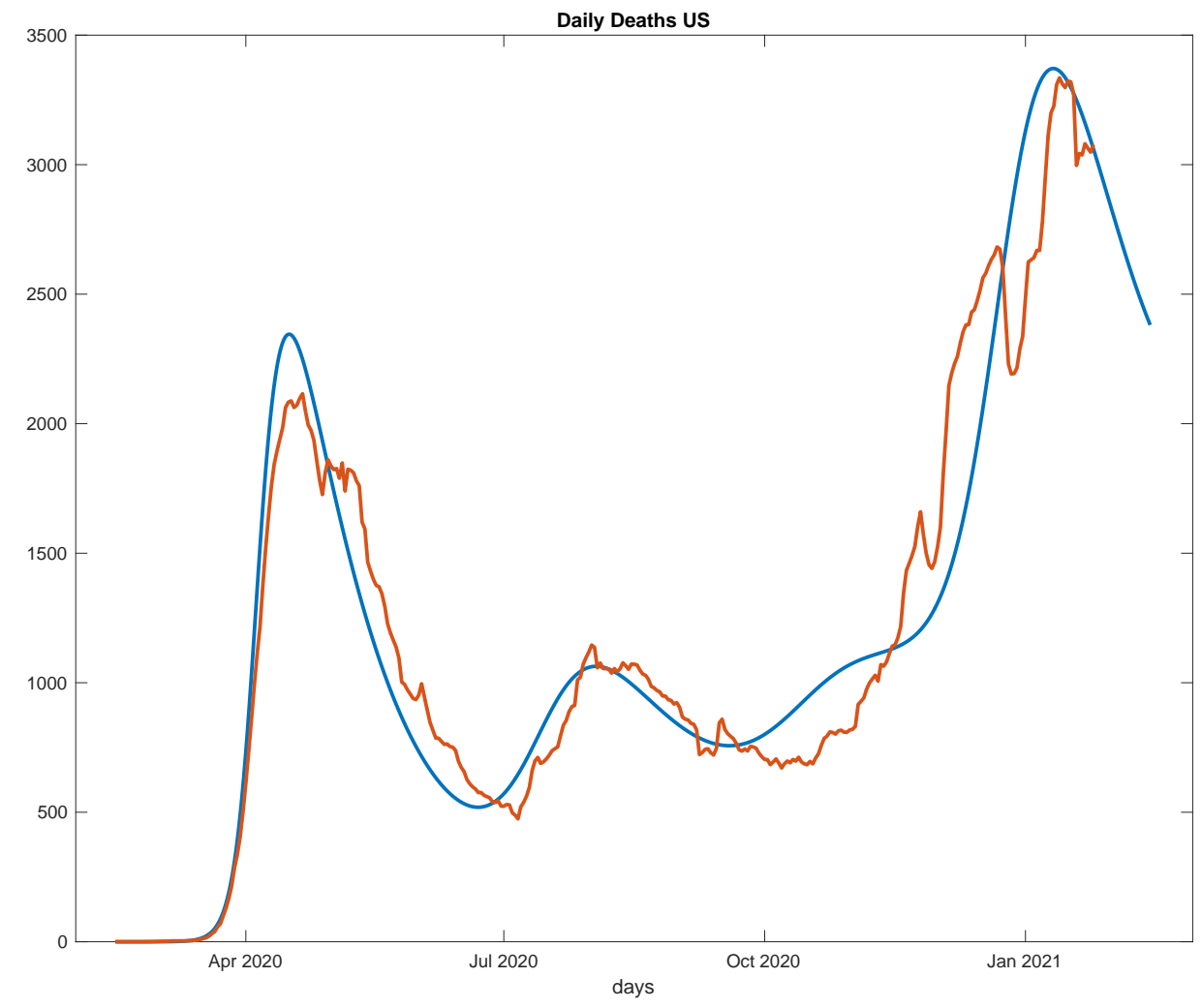

Figure 1: Daily Deaths Model (in blue) and Data (in red). The red line is a seven day moving average of daily deaths for the United States as reported on the COVID Tracking Project https: //covidtracking.com/data/download. 


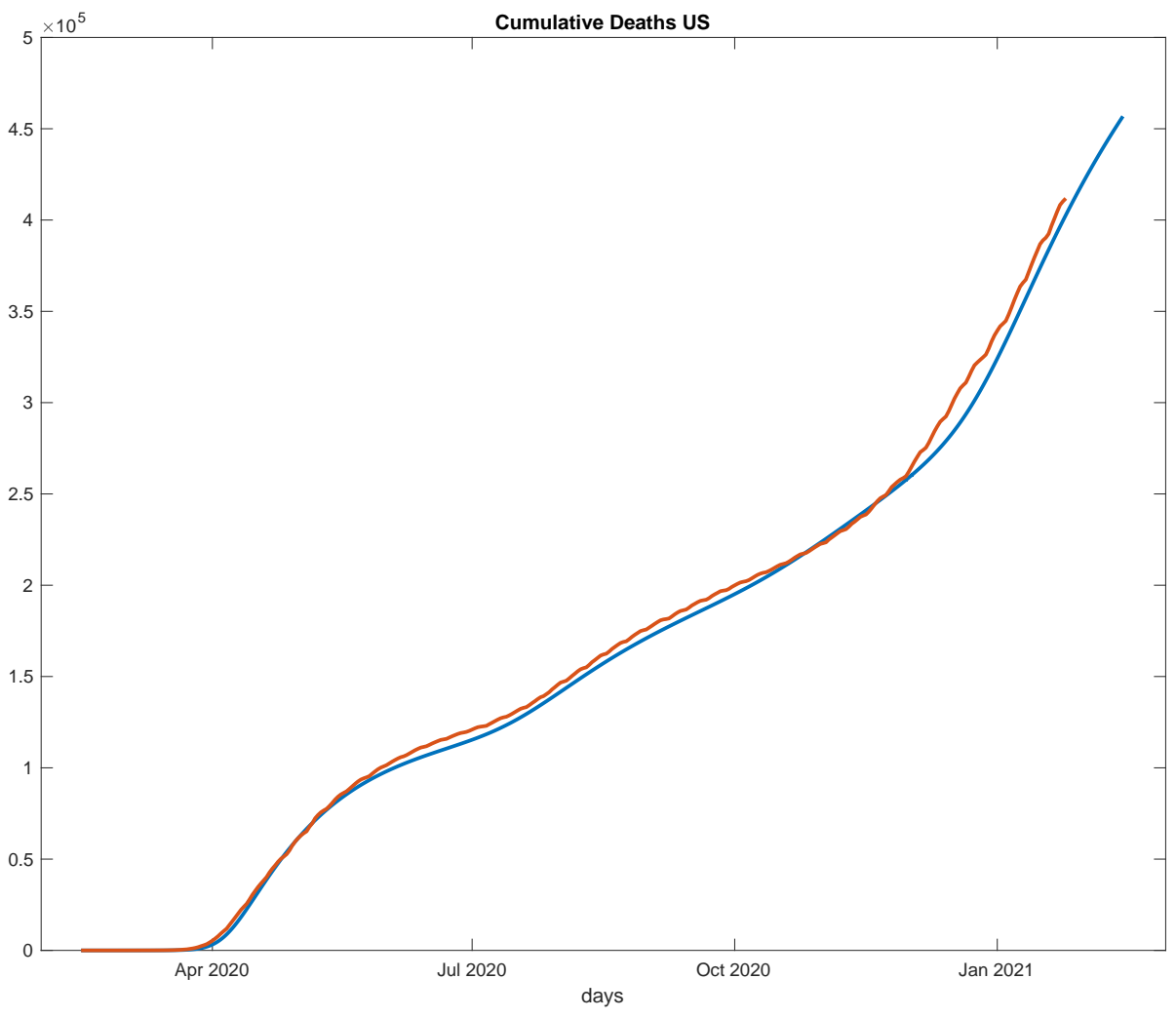

Figure 2: Cumulative Deaths Model (in blue) and Data (in red). The red line is cumulative COVID deaths for the United States as reported on the COVID Tracking Project https://covidtracking. $\mathrm{com} /$ data/download. 


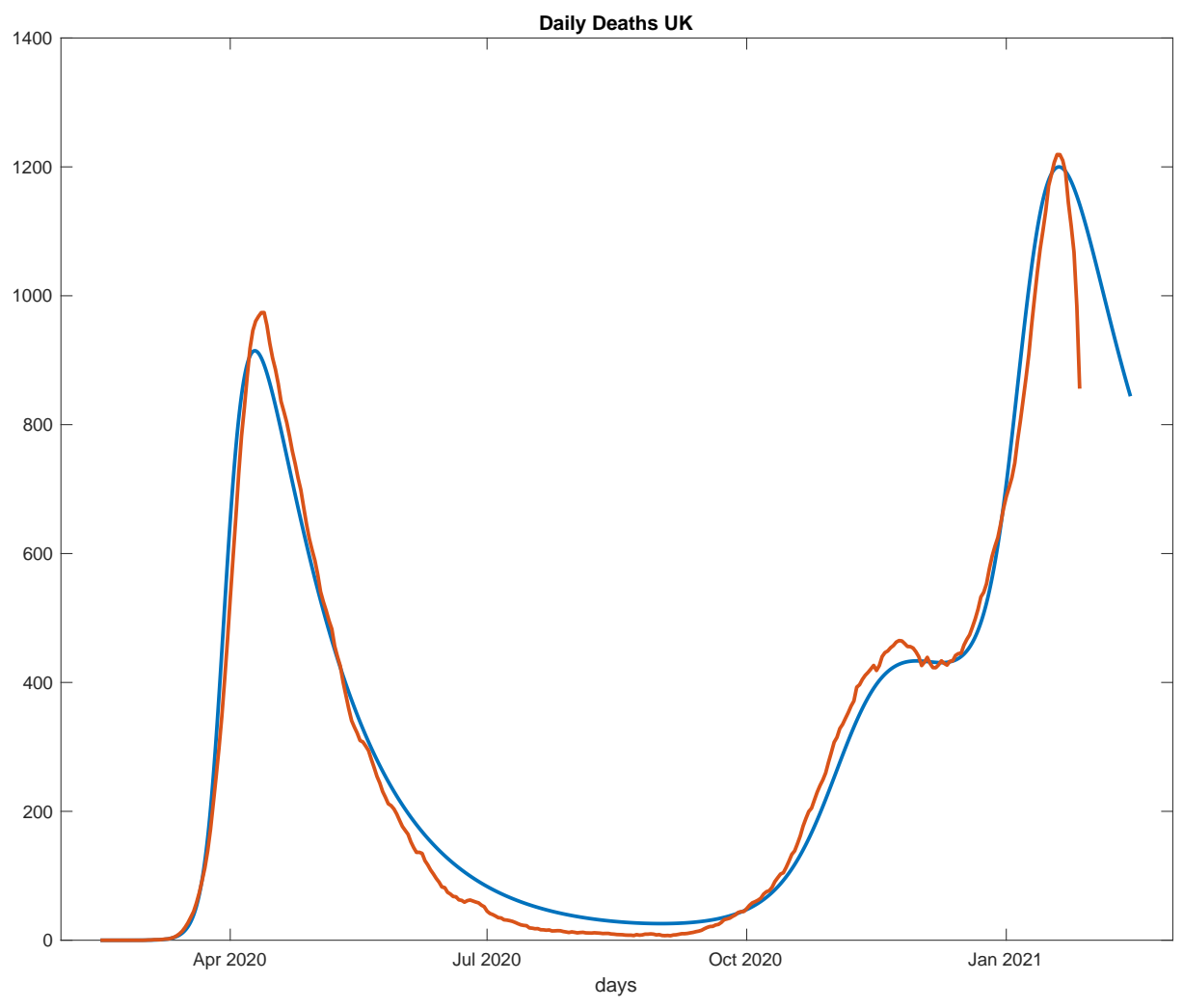

Figure 3: United Kingdom: Daily Deaths Model (in blue) and Data (in red). The red line is a seven day moving average of daily deaths for the the United Kingdom as reported on the GOV.UK site https://coronavirus.data.gov.uk/details/deaths. Deaths data are those Deaths within 28 days of positive test by date of death. 


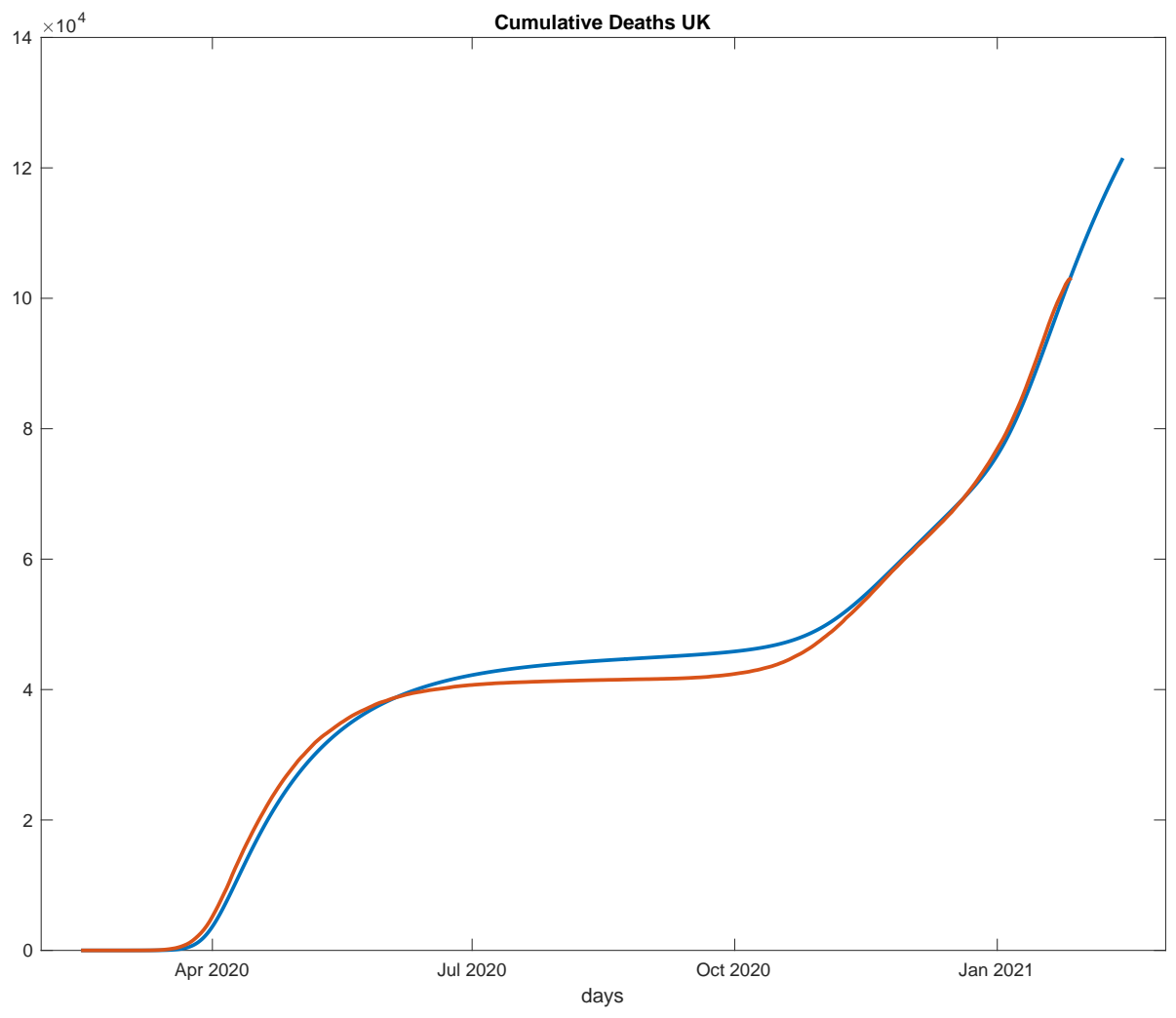

Figure 4: United Kingdom: Cumulative Deaths Model (in blue) and Data (in red). The red line is cumulative COVID deaths for the United Kingdom as reported on the GOV.UK site https: //coronavirus.data.gov.uk/details/deaths. Deaths data are those Deaths within 28 days of positive test by date of death.

I show the driving shocks for seasonality and pandemic fatigue for the United States, in Figures 5 and 6.

Figure 5 shows the basic reproduction number corresponding to no reduction in transmission due to a behavioral response (the transmisability of the virus with behavior at pre-pandemic patterns) for the United States. We see that the assumed pattern for seasonality in the US introduces a $35 \%$ reduction in transmissibility of the virus holding behavior fixed from the winter peak (centered on February 1) and 
the summer low. The seasonal pattern assumed for the UK is substantially larger I assume a ratio of peak to trough transmissibility holding behavior constant of 2.7 , with peak transmission occurring also on February 1. I also assume that at peak, transmission in the UK is $20 \%$ higher than in the US, holding behavior fixed (so the basic reproduction number in the UK peaks at 3.6 rather than 3).

Figure 6 shows the ratio of the semi-elasticity of the transmission rate with respect to the level of daily deaths relative to its initial level for the United States. We see in that figure that this semi-elasticity is assumed to fall to $37.5 \%$ of its original level late in the year. For the United Kingdom, the assumed decline of this semi-elasticity due to pandemic fatigue is smaller (it falls to $50 \%$ of its initial level) and it occurs with similar timing (ten days later in the UK). Note that the baseline value for the semi-elasticity of transmission with respect to deaths in the United Kingdom is assumed to be only $56 \%$ of the value for the United States.

For the United Kingdom, we model the introduction of a new variant of the COVID virus as the introduction of one exposed individuals with the new virus on September 1, 2020. This new variant is modeled as having the same seasonal pattern in transmissibility and to be subject to the same proportional reduction in transmissibility due to behavior as the original variant. But, holding the date and behavior constant, the new variant is assumed to be $38 \%$ more transmissible than the original variant. In Figure 7, I show the model's implication for the fraction of the currently infected population that are infected with the new variant. In this figure, we see that this new variant began to appear in measurable numbers in November and accounted for $70 \%$ of total infections by the end of 2020. This fraction matches data on the fractions of those tested in the UK revealed to have the new variant available in Figure 2 of the linked document on the new variant from the European Center for Disease Prevention and Control. https://www.ecdc.europa.eu/sites/default/files/documents/ COVID-19-risk-related-to-spread-of-new-SARS-CoV-2-variants-EU-EEA-first-update. pdf 


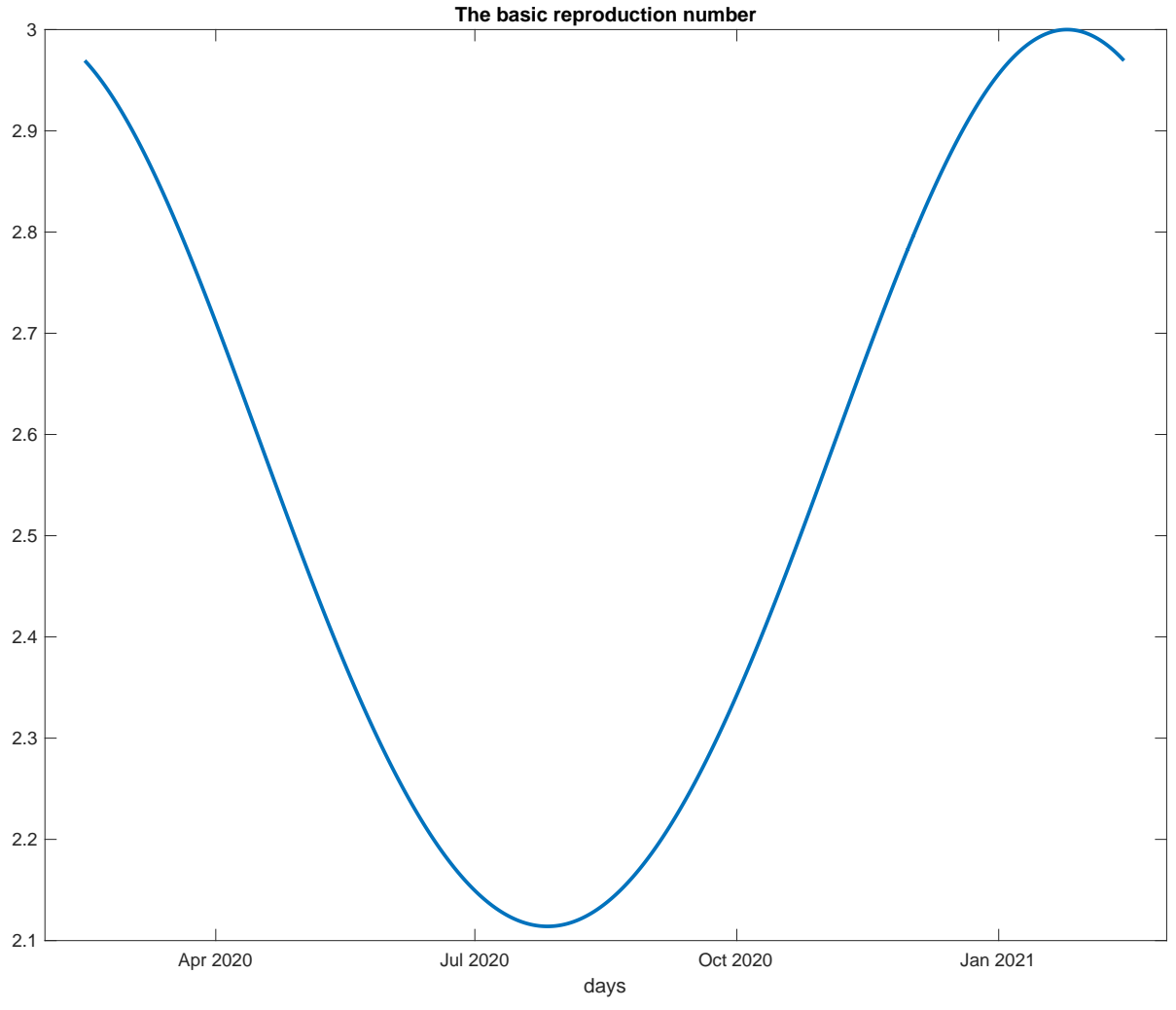

Figure 5: Assumed seasonality in the basic reproduction number. Peak transmission is on February 1 . 


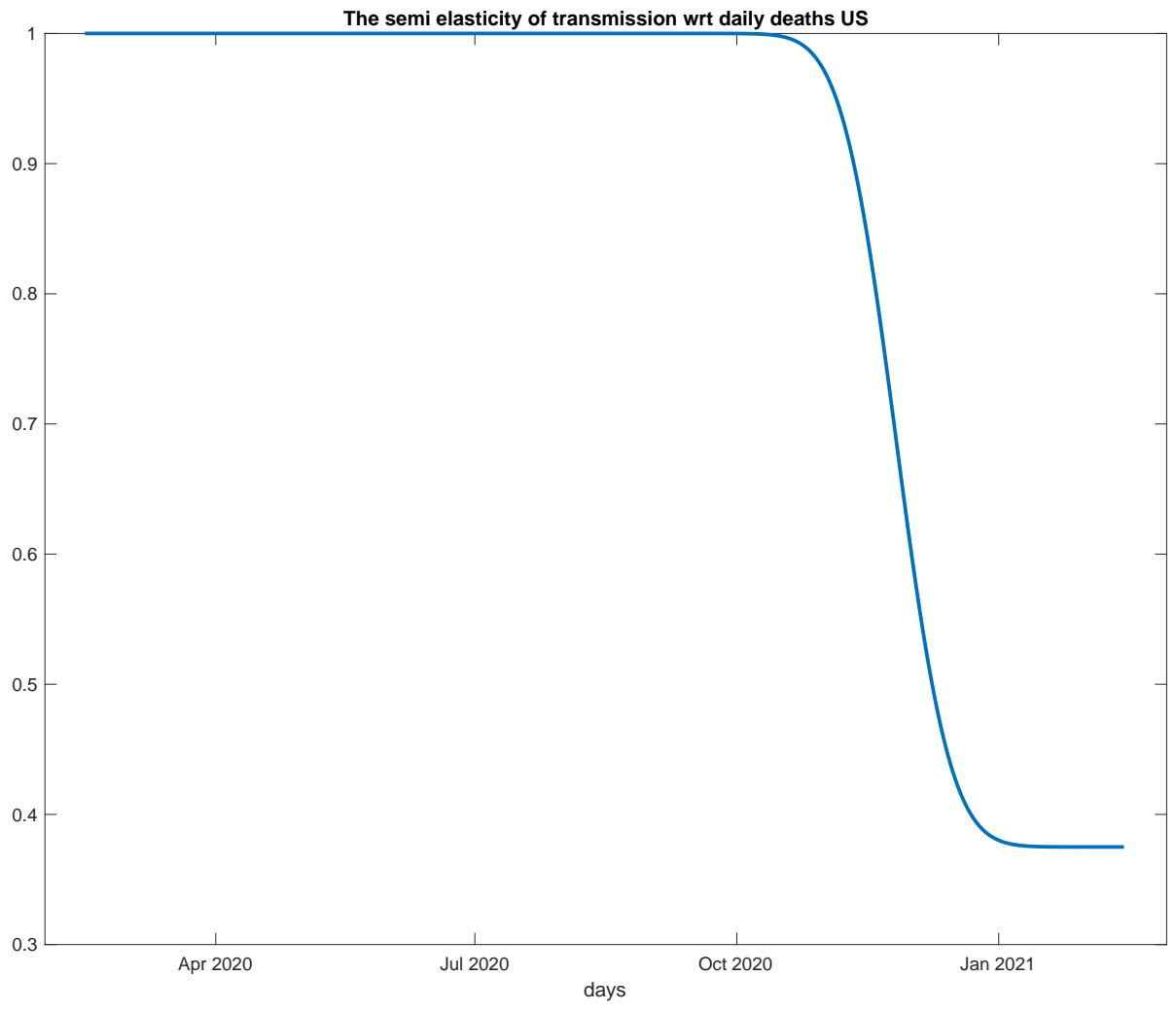

Figure 6: Assumed pandemic fatigue. The blue line shows the evolution of the semi-elasticity of transmission with respect to daily deaths relative to its initial level. 


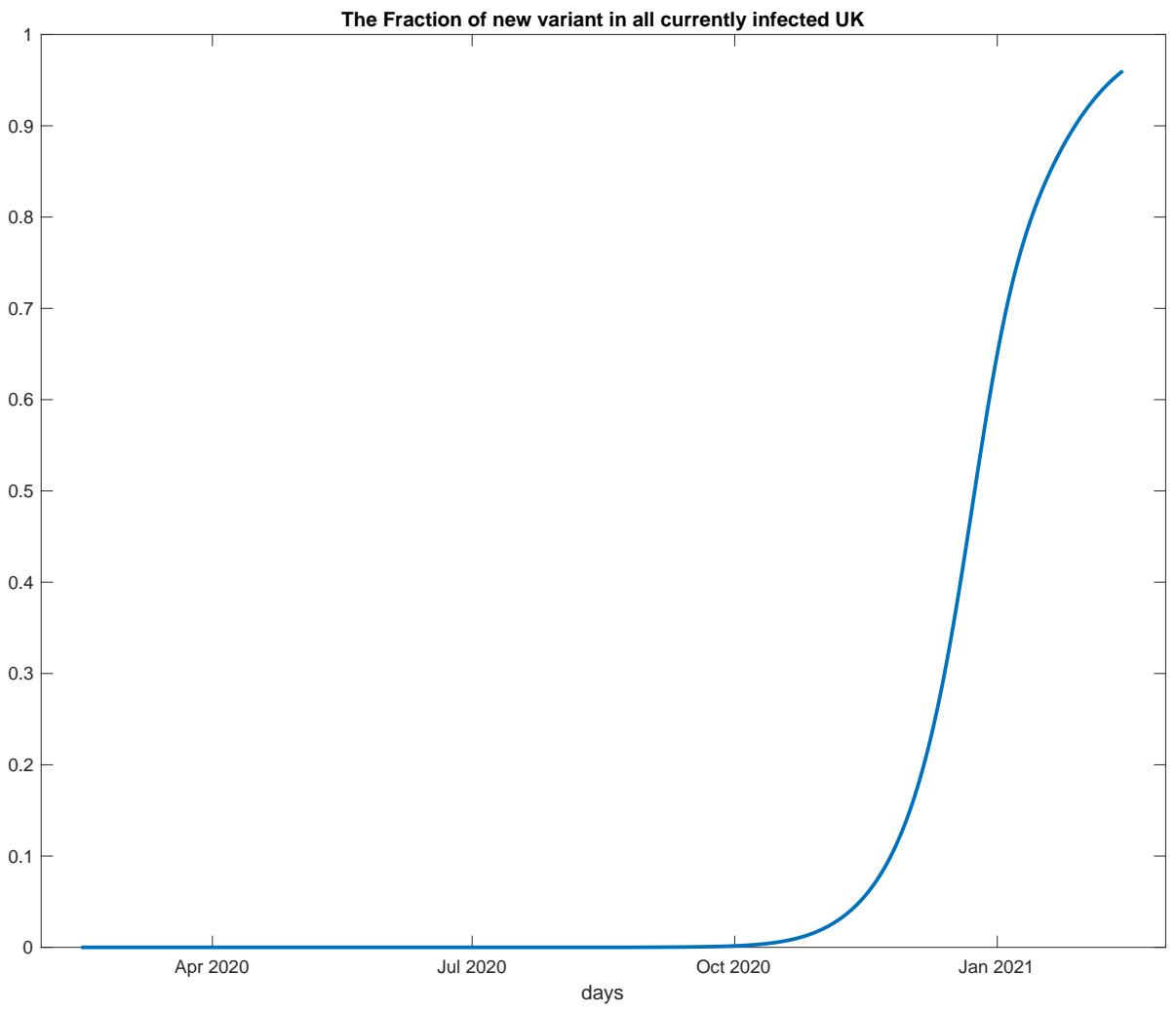

Figure 7: Model implication for fraction of total currently infected population infected with the new variant for the United Kingdom

\subsection{No seasonality nor pandemic fatigue}

Results for the United States and the United Kingdom for daily deaths for the first alternative simulation with neither seasonality nor pandemic fatigue are shown in Figures 8 and 9 . We can see in these figures that the predicted pandemic in this case is a poor fit to the data. Thus, I conclude that the two shocks together play an important role in shaping the evolution of the pandemic. We do, however, see the endogenous dynamics in the model in the multiple waves of deaths that occur in the 
model even in the absence of shocks. This was first pointed out in Cochrane (2020). ${ }^{9}$ As we shall see in our third alternative simulation, without these endogenous model dynamics, the model does a poor job in accounting for the daily deaths data even with the seasonality and pandemic fatigue shocks.

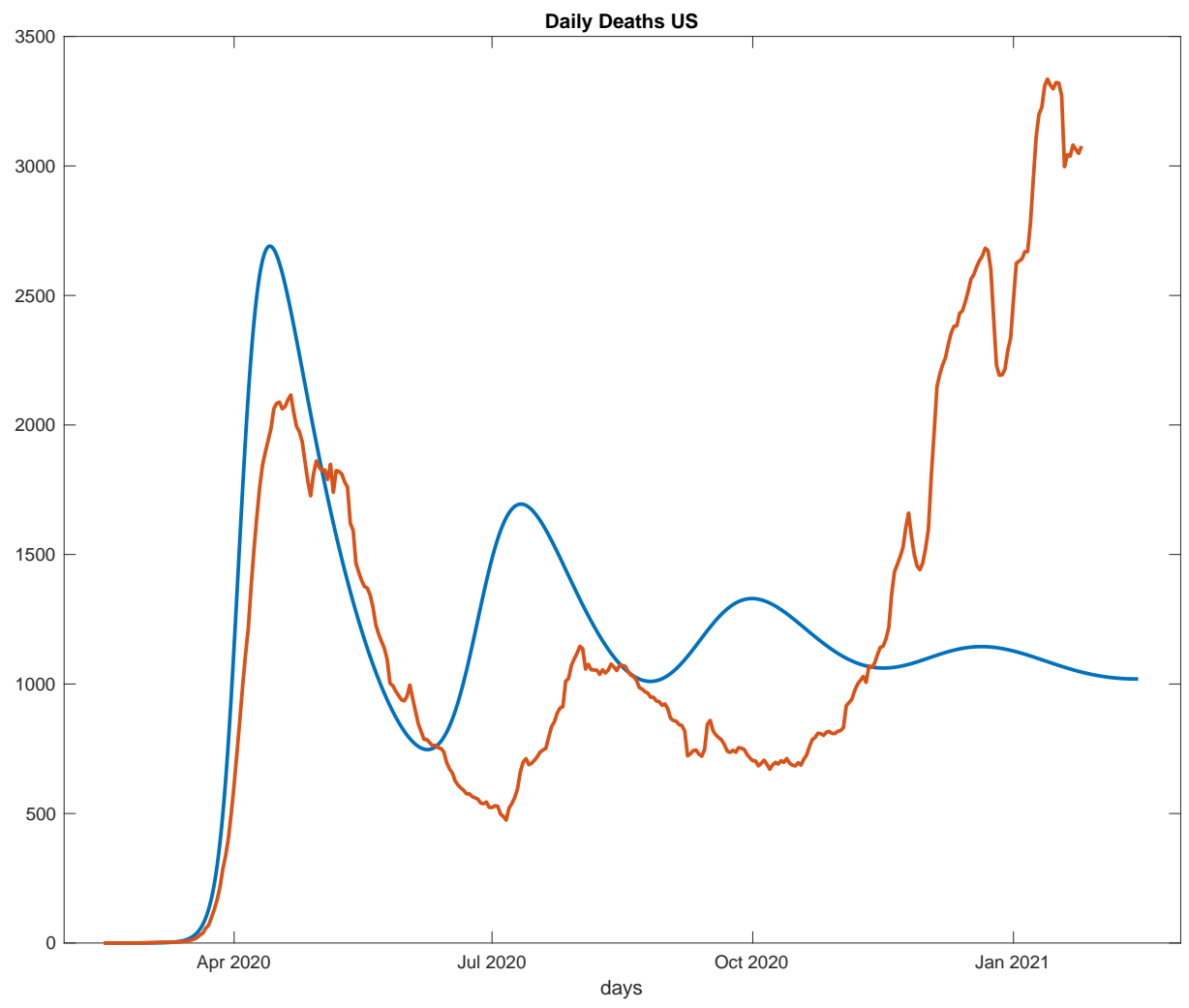

Figure 8: US Daily Deaths Model (in blue) and Data (in red) with neither the seasonality nor pandemic fatigue.

\footnotetext{
${ }^{9}$ See https://johnhcochrane.blogspot.com/2020/05/an-sir-model-with-behavior.html.
} 


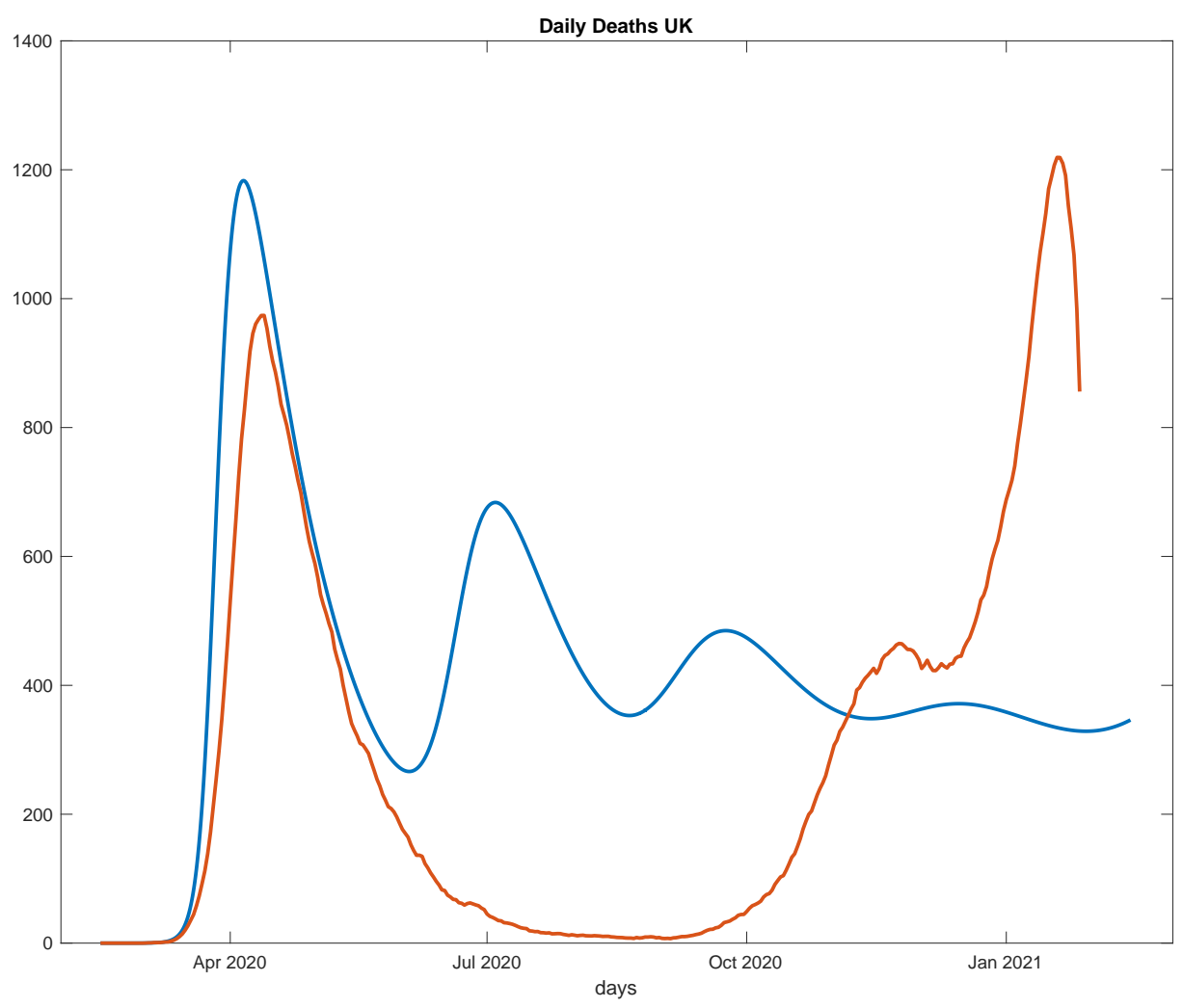

Figure 9: UK Daily Deaths Model (in blue) and Data (in red) with neither the seasonality nor pandemic fatigue.

\subsection{With seasonality but no pandemic fatigue}

Results for the United States and United Kingdom for daily deaths for the second alternative simulation with seasonality but no pandemic fatigue are shown in Figures 10 and 11. We can see in these figures that the predicted pandemic in this case matches the data quite well through the summer of 2020, but then the model completely misses the large wave of daily deaths in the late Fall and Winter. As discussed above, on the basis of these results I conclude that pandemic fatigue played an important role in driving this wave of deaths seen in both countries late in the 
year.

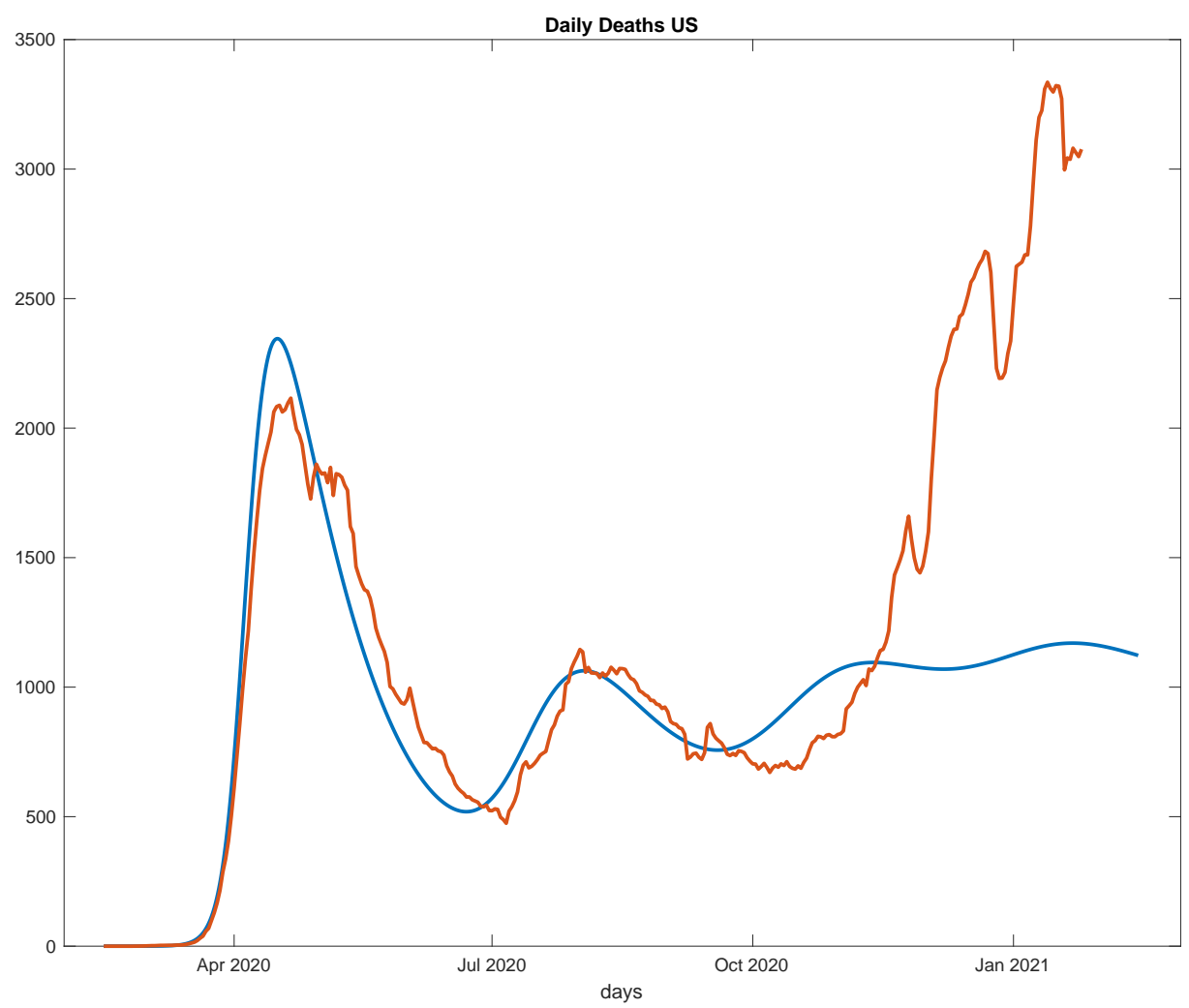

Figure 10: Daily Deaths Model (in blue) and Data (in red) with seasonality but no pandemic fatigue. 


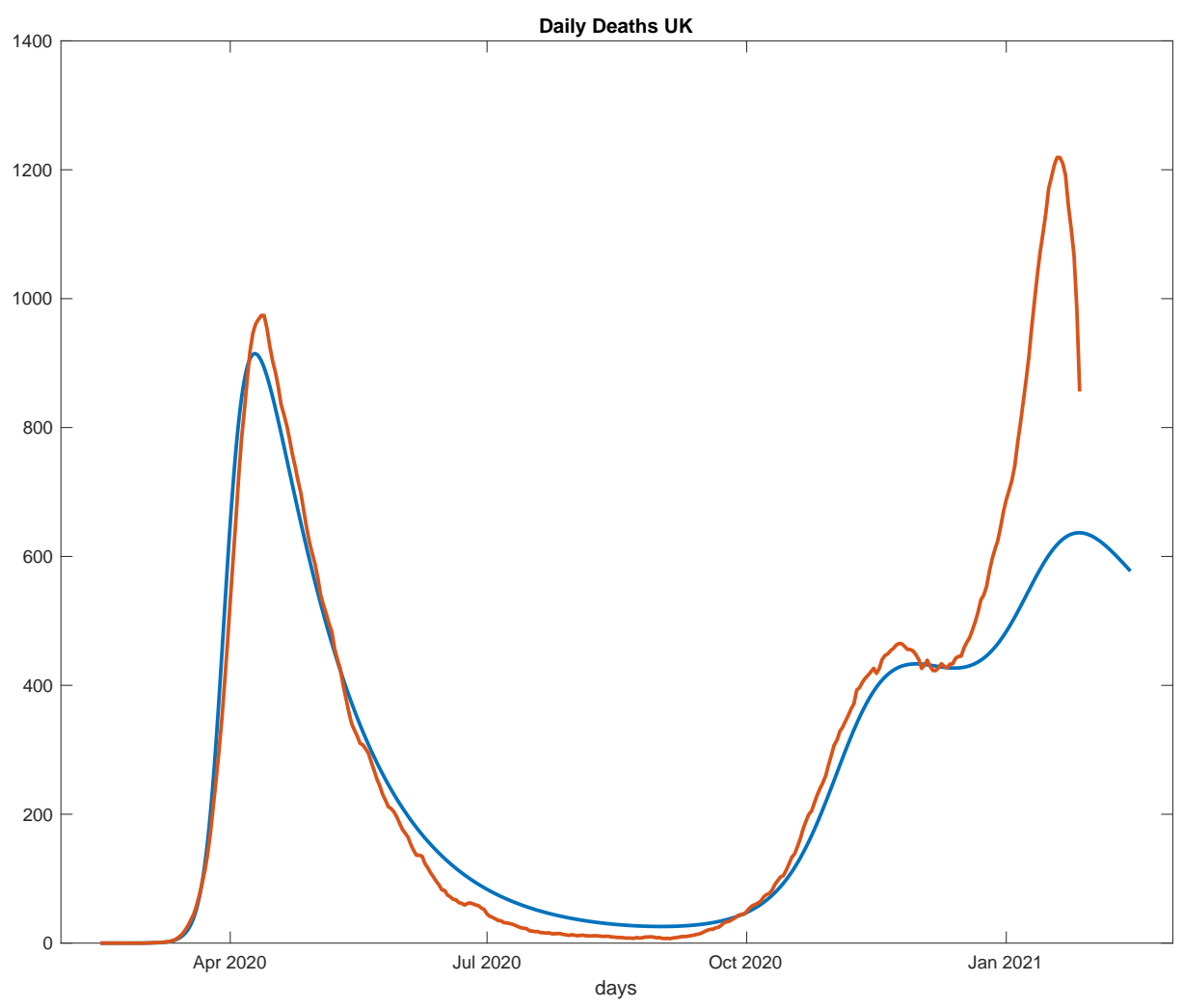

Figure 11: UK Daily Deaths Model (in blue) and Data (in red) with seasonality but no pandemic fatigue.

\section{With seasonality and pandemic fatigue, but only a short expected stay in $H$}

In my final alternative simulation, I include seasonality and pandemic fatigue, but I dramatically shorted the time agents expect to spend in compartment H. Specifically, I set the flow rate from compartment $\mathrm{H}$ to either recovery or death to $\zeta=10$, corresponding to an expected stay of only one tenth of a day. This is tantamount to eliminating this compartment. Results for daily deaths for the United States and 
the United Kingdom for this third alternative simulation are shown in Figures 12 and 13. We see in these figures that this alternative simulation fails to match the patterns seen in the initial large waves of deaths in the Spring and early Summer. By comparing these figures to Figures 1 and 3, we see that the long delay in compartment $\mathrm{H}$ is critical for allowing the model to match the early dynamics of the pandemic.

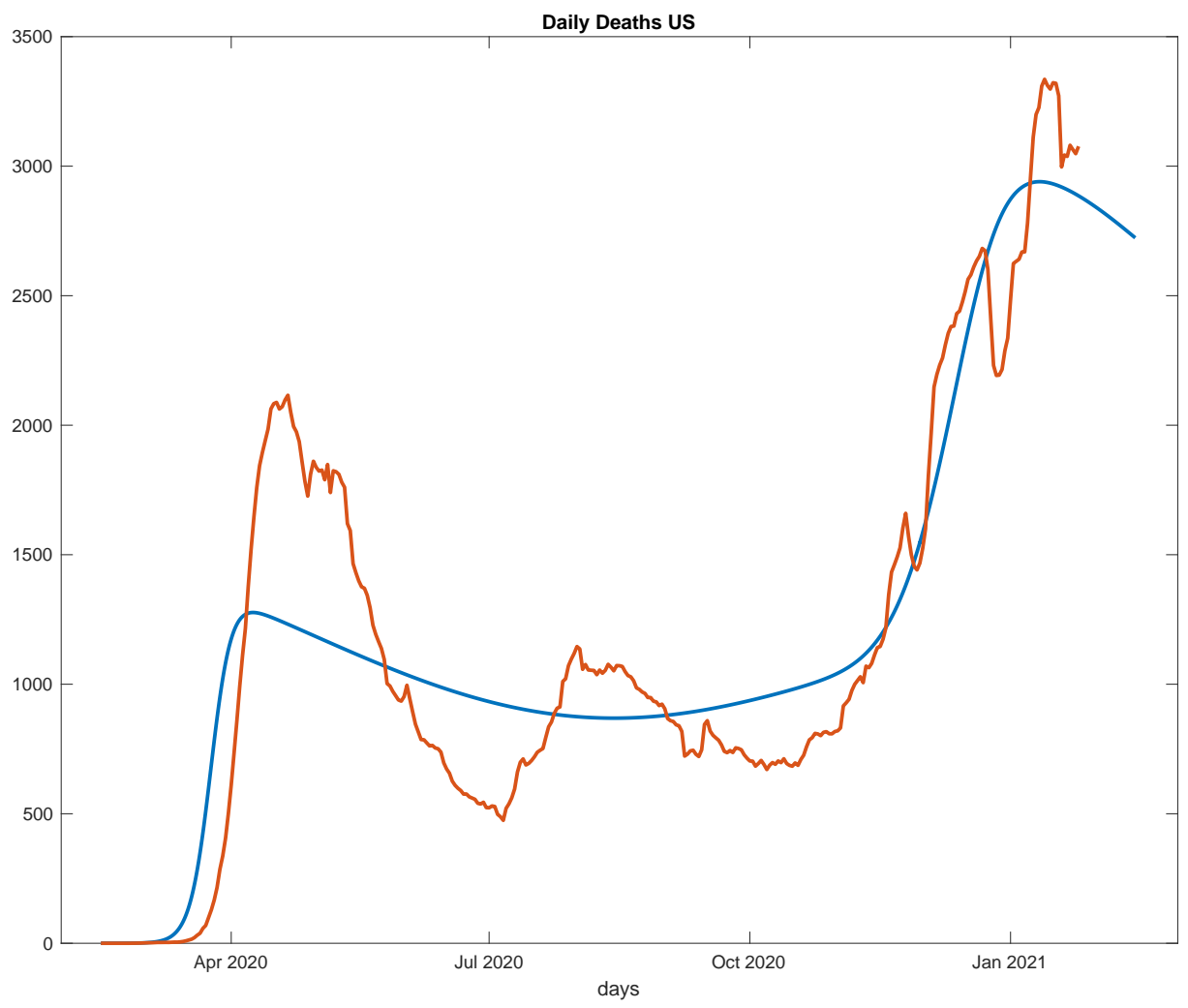

Figure 12: US Daily Deaths Model (in blue) and Data (in red) with seasonality and pandemic fatigue but a short expected time in the compartment $H$. 


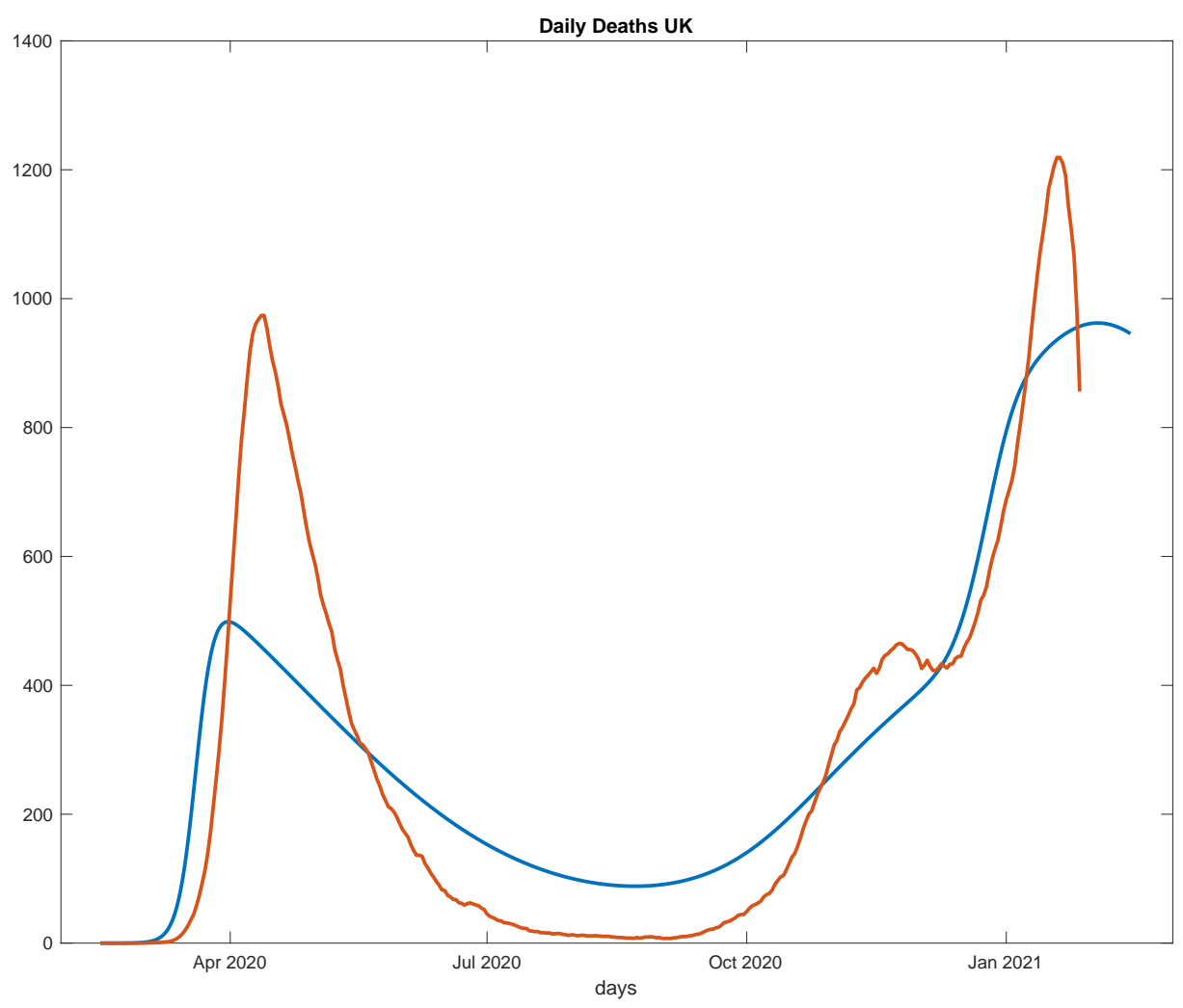

Figure 13: UK Daily Deaths Model (in blue) and Data (in red) with seasonality and pandemic fatigue but a short expected time in the compartment $H$.

To relate the results here directly to those in Atkeson, Kopecky, and Zha (2021), I show the model implied growth rate of daily deaths for the US with and without a long stay in compartment $\mathrm{H}$ in Figures 14 and 15. In both of these figures, we see that behavior causes the growth rate of daily deaths to decline rapidly from very high levels (the y-axis in both figures is truncated at 30\%) to levels close to zero very rapidly early on in the pandemic. Thus, in either specification of the model, the first peak of daily deaths is much lower than would be the case in a standard model with no behavioral response of transmission to the level of daily deaths. But in comparing Figures 14 and 15, we see that the delayed behavioral response of transmission to 
increasing new infections allows for fluctuations in the growth rate of daily deaths in a range about 6 or 7 percentage points wide, while the model with the short stay in compartment $\mathrm{H}$ does not allow for such fluctuations. In this sense, the behavioral response is too strong in the model with only a short stay in compartment $\mathrm{H}$ relative to the variation in growth rates of daily deaths observed in the data.

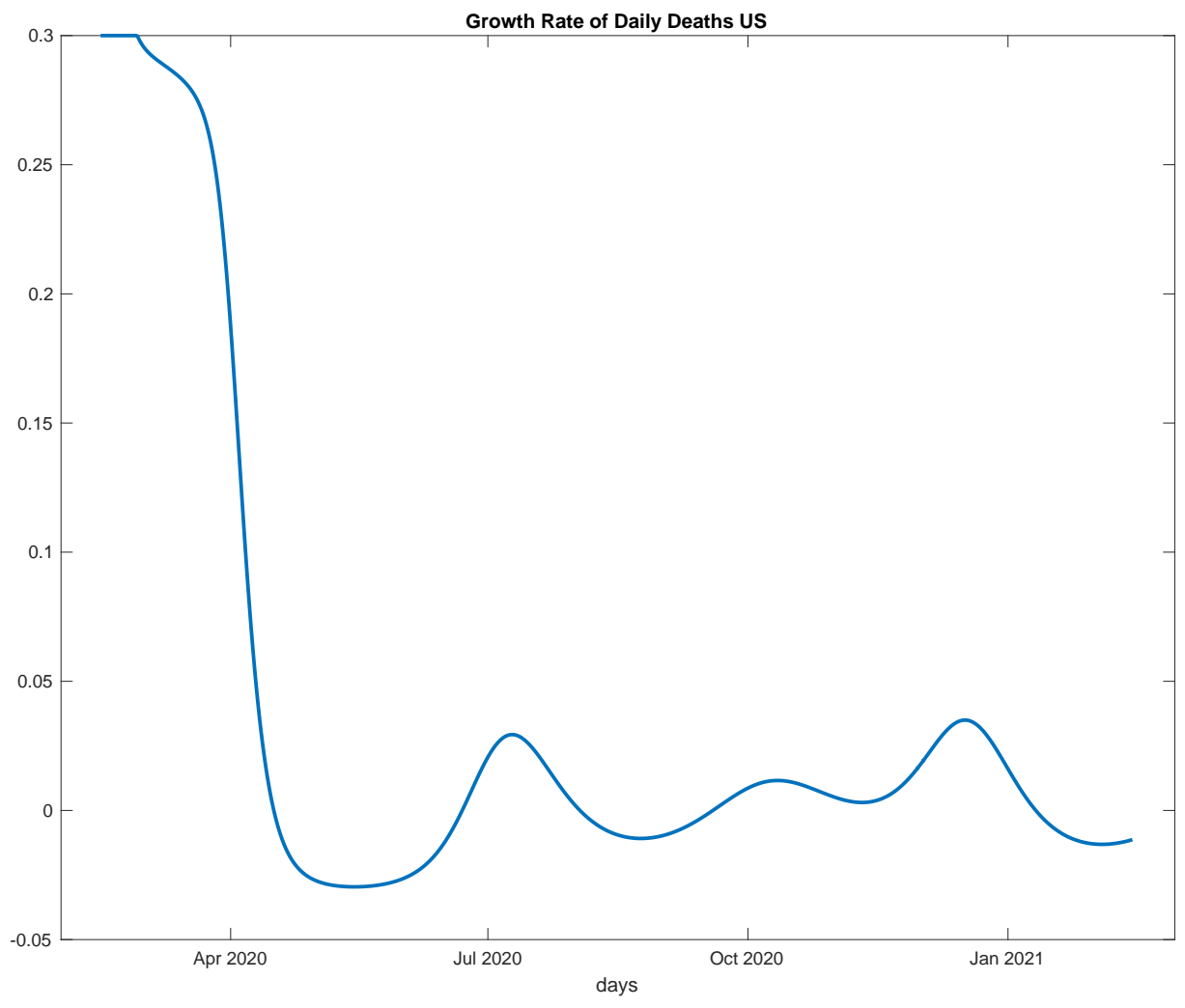

Figure 14: US model implied growth rate of Daily Deaths in the baseline simulation with a long expected stay in compartment $H$ 


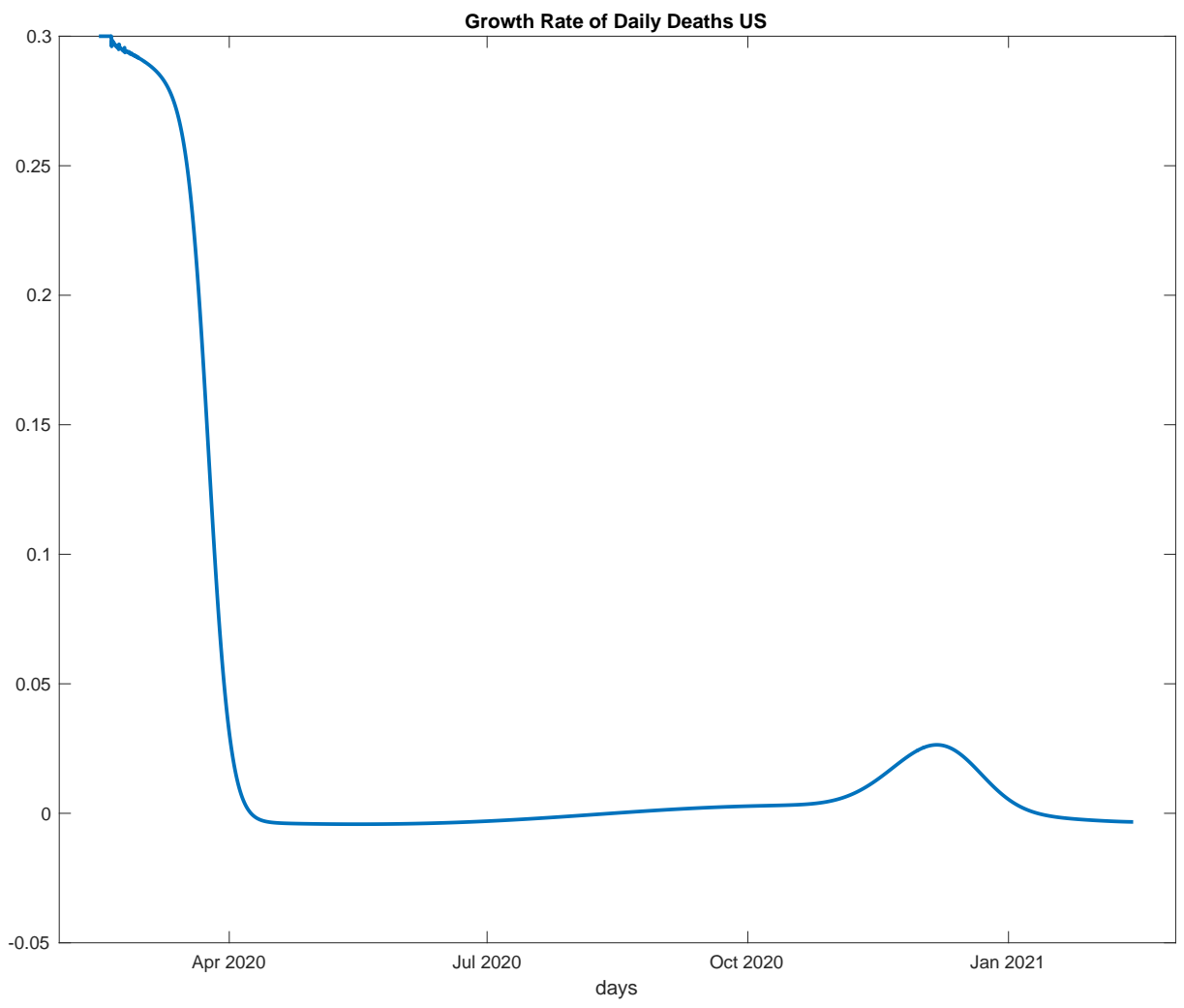

Figure 15: US model implied growth rate of Daily Deaths in the baseline simulation with a short expected stay in compartment $H$

\section{Model Forecast for 2021 and 2022}

I now use the model to generate forecasts for the evolution of the epidemic over a three year period from February 15, 2020 to February 15, 2023. In this forecast, I assume that the seasonality of transmission continues and that the semi-elasticity of transmission with respect to daily deaths remains at its new lower level for the remainder of the epidemic (i.e. pandemic fatigue continues). I also introduce the new, more transmissible, variant to the United States on December 1, 2020. At 
this point, I do not include consideration of vaccines. These forecasts might then be considered a baseline against which progress on vaccines and/or improvements in policy might be evaluated.

Results for the United States for daily and cumulative deaths for the forecast simulation are shown in Figures 16 and 17. In those figures, we see that the model predicts a substantial decline in daily deaths in the early part of 2021 with a large third wave in the late Spring of that year followed by a disappearance of the disease in late 2022. This large third wave of deaths is driven by the new, more contagious variant. The model predicts that this variant will account for $100 \%$ of all current infections by summer of 2021. We also see that cumulative deaths from the disease are forecast to asymptote to 1.25 million as the epidemic comes to an end.

In Figure 18 I show the model's implications for the fraction of the population that has experienced infection at each date over the period from February 15, 2020 through February 2023. We see the this fraction of the population asymptotes to $75 \%$ as the epidemic comes to an end..$^{10}$

The corresponding forecasts for the United Kingdom are shown in Figures 19, 20, and 21. The model forecasts a large third wave of deaths in the United Kingdom in late 2021 and into early 2022. The long run number of deaths is forecast at just over 240,000. The cumulative fraction of the population ever infected asymptotes to a level just over $70 \%$.

\footnotetext{
${ }^{10}$ Note that this fraction does not converge to the level implied by the basic reproduction number of 5 for the new variant at its Winter peak transmissibility (that fraction would be $80 \%$ ) because of the seasonality in transmissibility.
} 


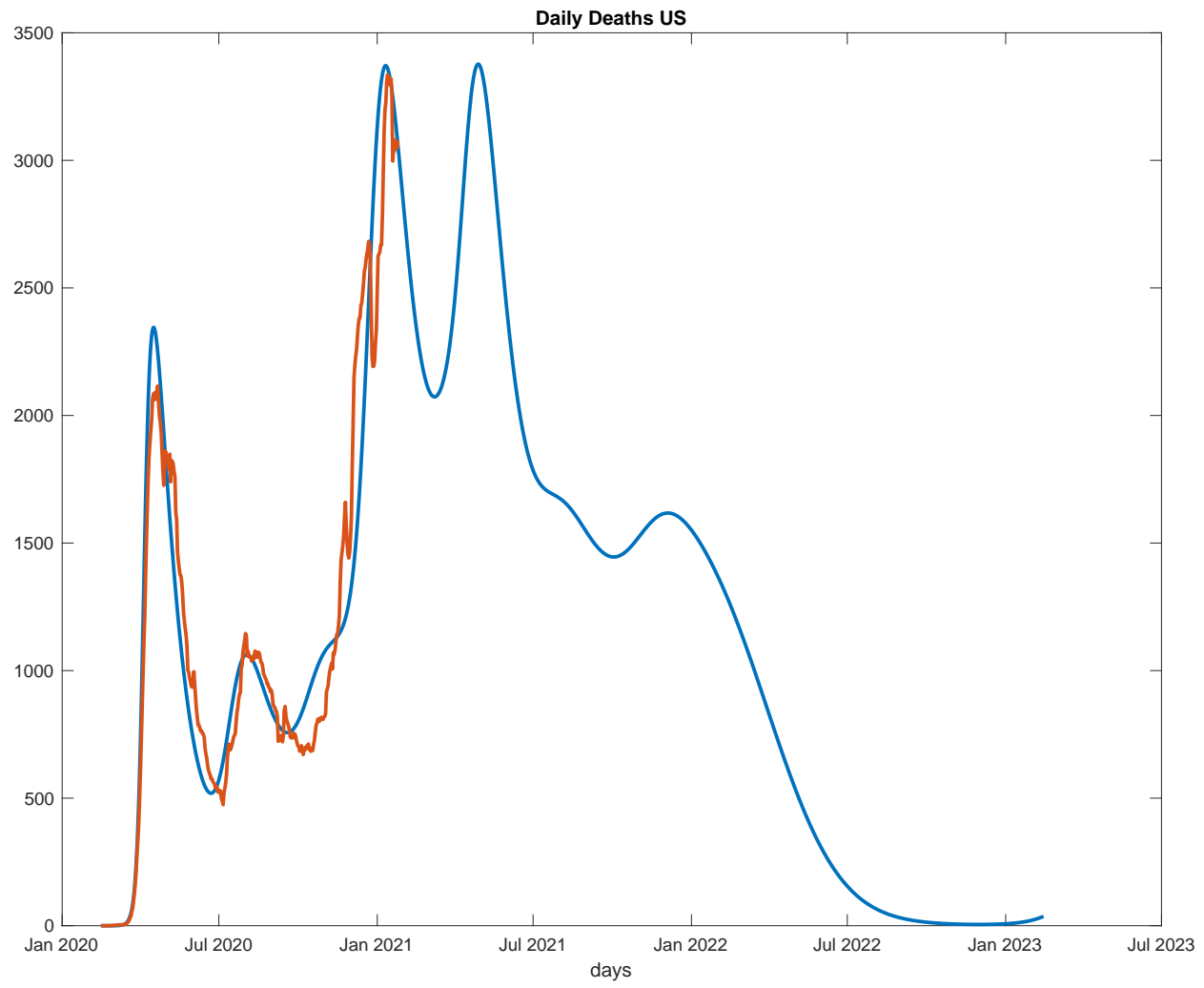

Figure 16: US Forecasts for the epidemic over three years from Feb 15, 2020 to Feb 15 2023. Daily Deaths Model (in blue) and Data (in red). 


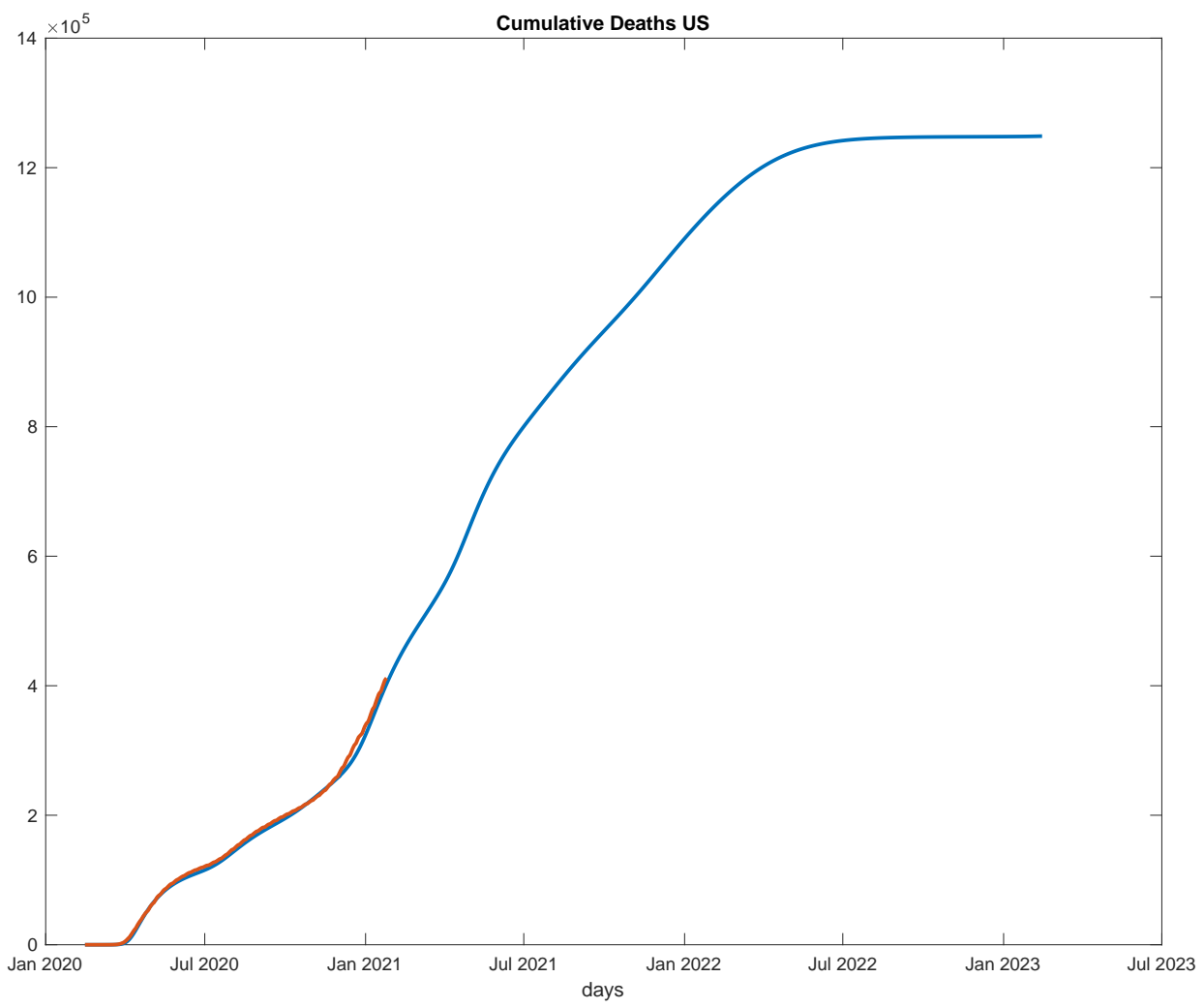

Figure 17: US Forecasts for the epidemic over three years from Feb 15, 2020 to Feb 15, 2023. Cumulative Deaths Model (in blue) and Data (in red). 


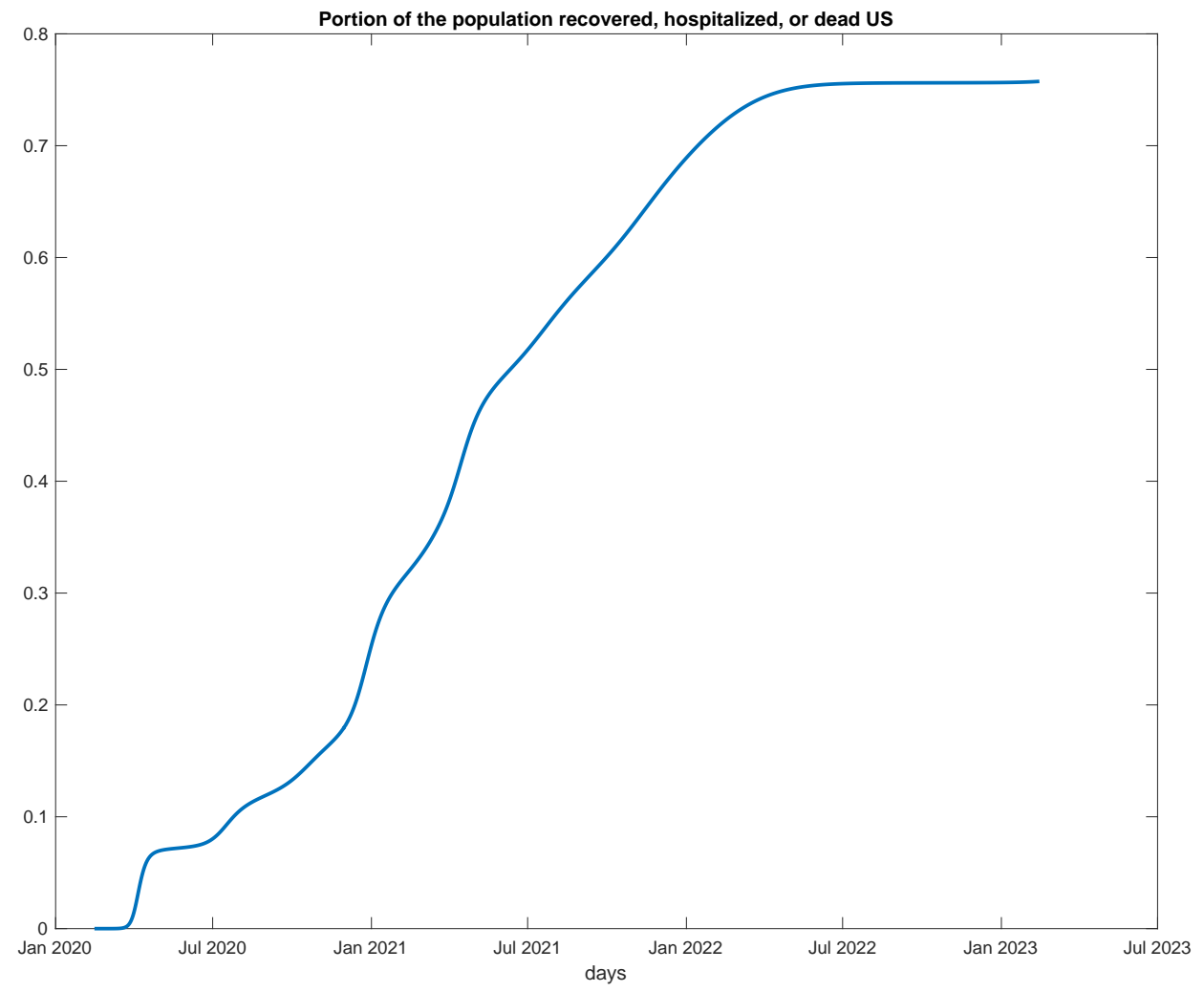

Figure 18: US Forecasts for the epidemic over three years from Feb 15, 2020 to Feb 15 2023. The blue line in this figure shows the fraction of the population that has ever been infected with the disease at each date. This fraction is the sum of the fractions of agents in compartments $R, H$, and $D$. 


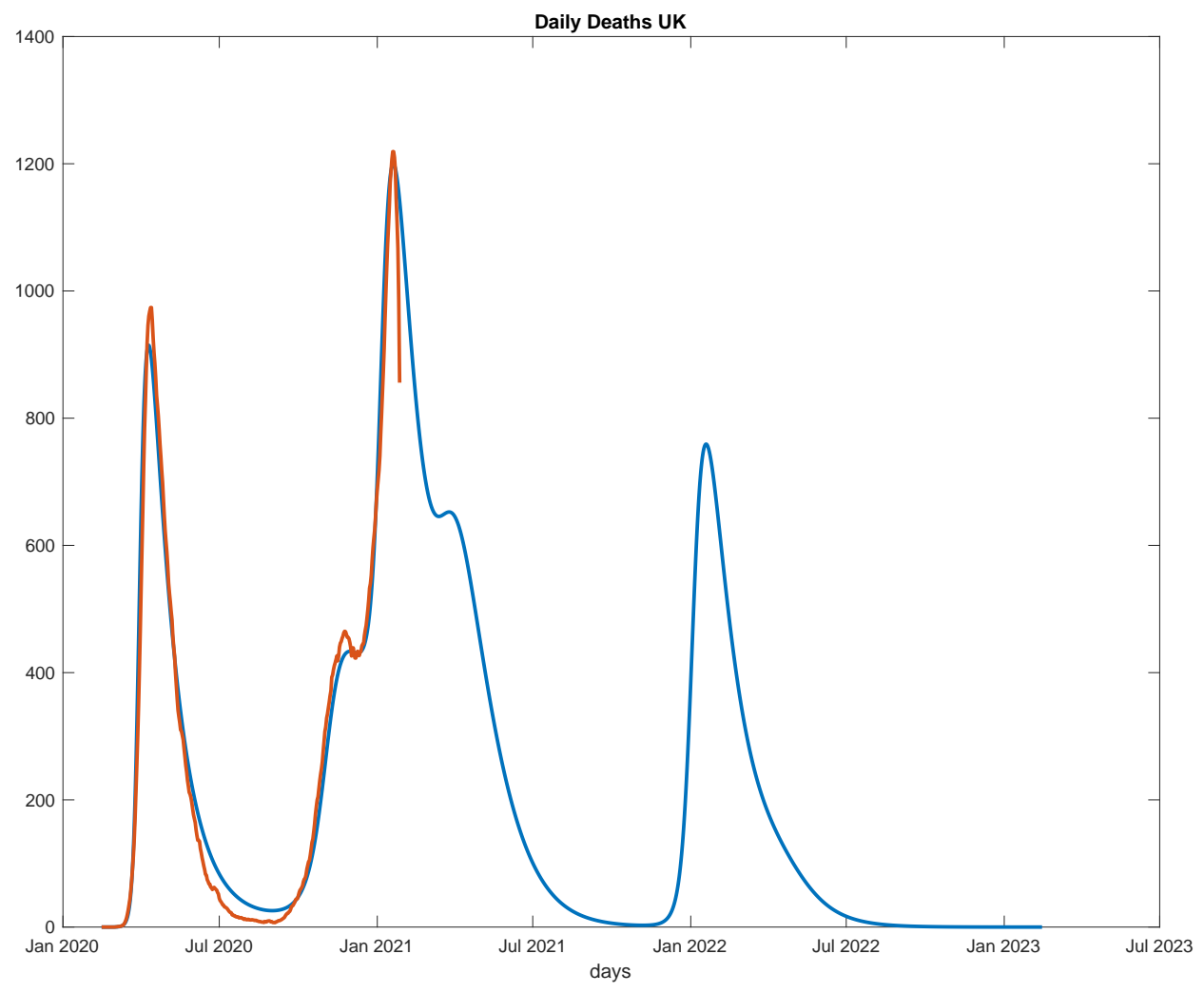

Figure 19: UK Forecasts for the epidemic over three years from Feb 15, 2020 to Feb 152023. Daily Deaths Model (in blue) and Data (in red). 


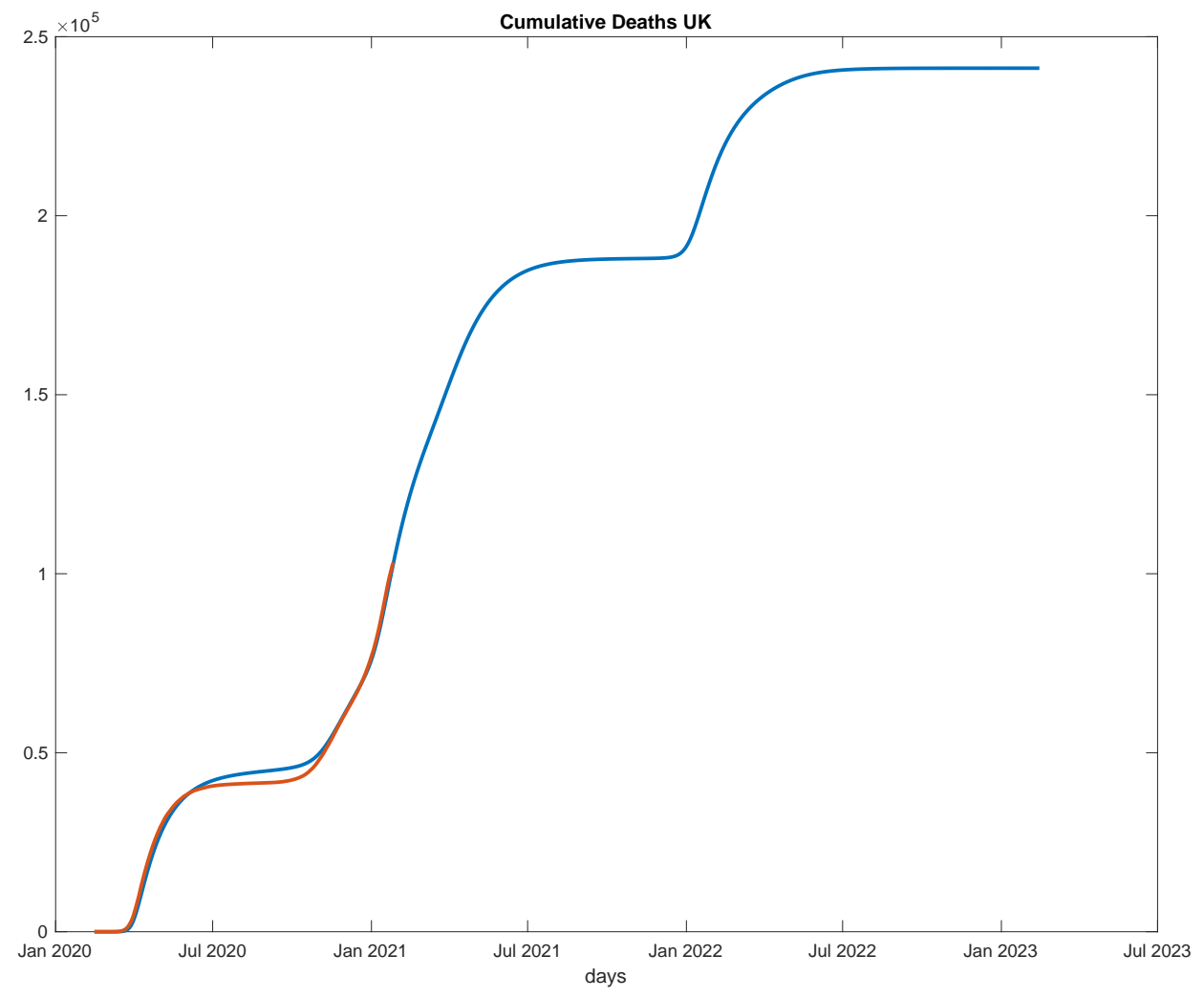

Figure 20: UK Forecasts for the epidemic over three years from Feb 15, 2020 to Feb 15, 2023. Cumulative Deaths Model (in blue) and Data (in red). 


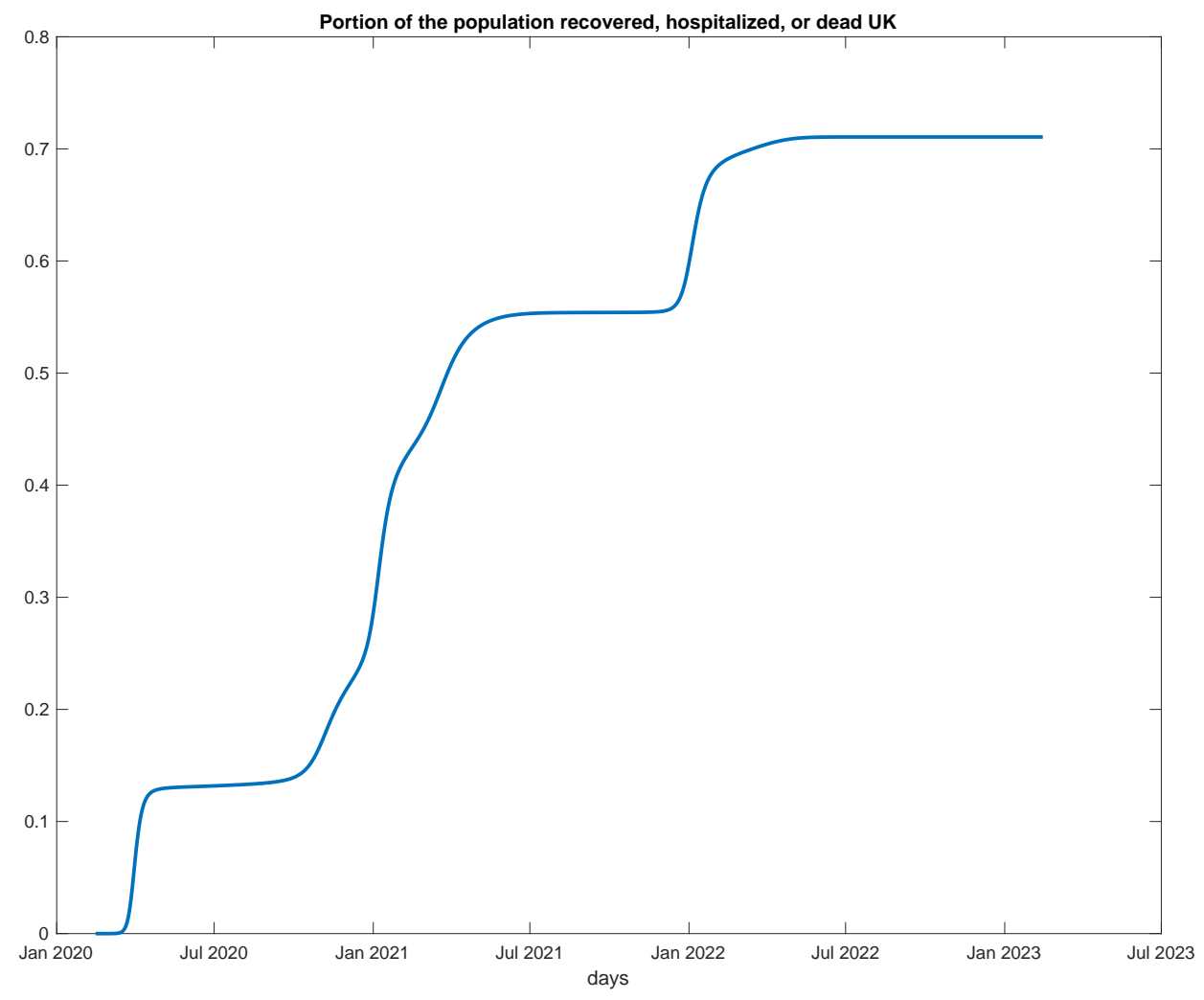

Figure 21: UK Forecasts for the epidemic over three years from Feb 15, 2020 to Feb 15 2023. The blue line in this figure shows the fraction of the population that has ever been infected with the disease at each date. This fraction is the sum of the fractions of agents in compartments $R, H$, and $D$.

\section{Discussion}

In fitting the model considered here to the data, I have chosen the parameters governing behavior and the seasonal and pandemic fatigue shocks by a process of trial and error. The model is highly non-linear, and, as a result, estimation of these parameter through some more formal method may prove daunting. In future work, however, I would like to develop some results regarding the identification of parameters and 
shocks from a data fitting exercise. Is the seasonal variation in transmission consistent with other data (it does line up with the timing of the seasonality of influenza in these two countries)? Is there some other specification of the model that would reduce the need to introduce pandemic fatigue to account for the the large wave of deaths seen in both countries late in the year? I do not know the answers to these questions at this time.

While the fit between this highly aggregated model and national deaths data shown here is remarkable, it is clear that this model leaves out many important details of the evolution of the COVID-19 epidemic in both the United States and the United Kingdom over the course of 2020. There was considerable geographic heterogeneity in the evolution of the epidemic at subnational levels and wide heterogeneity in the transmission and mortality impact of the virus across different demographic groups. Moreover, there is considerable evidence that the average infection fatality rate of the virus has been reduced over the course of the past year.

Given this important heterogeneity and additional shocks observed in the data, I see this modeling exercise as a proof of concept that incorporating a simple model of the endogenous behavioral response of the population (either through private behavior or policy) to disease prevalence can be used to account for and potentially forecast the evolution of a major epidemic. I see the development of models that combine realistic geographic and demographic heterogeneity and additional observed shocks with behavior as a task for future research.

The modeling of behavior in this paper is reduced-form. Structural models of behavior would model the costs and benefits to utility maximizing agents of various types of activity during a pandemic. Such models would also make contact with data on individual activity. Again, this is a task I leave for future research.

Finally, because this model of behavior is reduced-form, I cannot determine whether the modeled behavioral response of transmission to the level of daily deaths was the result of government mandates on activity imposed as a function of the prevalence of the disease or simply as a result of decentralized choices by individual agents. This 
is an important question for future research.

\section{Model and Parameters}

The model is as follows.

The SEIHR model extends the SIR model by adding both the exposed state $E$ and the hospitalized state $H$. In this version of the model the total population $N$ is

given by the sum of susceptible agents in state $S$, exposed in state $E$, infected in $I$, hospitalized in $H$, recovered in $R$, and dead in $D$.

To model the introduction of a new variant, I add separate compartments $E_{v}$ and $I_{v}$ for those exposed to and infectious with the new variant. The transmission rate of the original variant is denoted by $\beta(t)$. That for the new variant is denoted by $\beta_{v}(t)$.

The dynamics of the model are given by

$$
\begin{gathered}
\frac{d S(t)}{d t}=-\left(\beta(t) I(t)+\beta_{v}(t) I_{v}(t)\right) S(t) \\
\frac{d E(t)}{d t}=\beta(t) I(t) S(t)-\sigma E(t) \\
\frac{d E_{v}(t)}{d t}=\beta_{v}(t) I_{v}(t) S(t)-\sigma E_{v}(t)+\bar{E}_{v}(t) \\
\frac{d I(t)}{d t}=\sigma E(t)-\gamma I(t) \\
\frac{d I_{v}(t)}{d t}=\sigma E_{v}(t)-\gamma I_{v}(t) \\
\frac{d H(t)}{d t}=\eta \gamma\left(I(t)+I_{v}(t)\right)-\zeta H(t) \\
\frac{d R(t)}{d t}=(1-\nu) \zeta H(t)+(1-\eta) \gamma\left(I(t)+I_{v}(t)\right)-\bar{E}_{v}(t)
\end{gathered}
$$




$$
\frac{d D(t)}{d t}=\nu \zeta H(t)
$$

The reduced-form for the behavioral response of the transmission rate to the level of daily deaths is given by

$$
\begin{aligned}
& \beta(t)=\bar{\beta} \exp \left(-\kappa(t) \frac{d D(t)}{d t}+\psi(t)\right) \\
& \beta_{v}(t)=\bar{\beta}_{v} \exp \left(-\kappa(t) \frac{d D(t)}{d t}+\psi(t)\right)
\end{aligned}
$$

where the parameters $\bar{\beta}$ and $\bar{\beta}_{v}$ control the baseline transmissibility of the normal and variant of COVID, the parameter $\psi(t)$ is used to introduce seasonality in transmission, and $\kappa(t)$ is the semi-elasticity of transmission with respect to the level of daily deaths.

The new variant is introduced by setting $\bar{E}_{v}(t)=1$ for one day on a specified date $t_{v}$ and equal to zero otherwise. Note that this quantity is subtracted off of the change in the $R$ compartment simply to keep the population constant. Since this shift is only one person for one day, it does not impact the quantitative implications of the model for large populations.

To model seasonality in the transmission of the virus, we set

$$
\psi(t)=\text { seasonalsize } *(\cos ((t+\text { seasonalposition }) * 2 \pi / 365)-1) / 2
$$

where seasonalsize controls the magnitude of the seasonal fluctuations in transmissibility holding behavior fixed and seasonalposition controls the location of the seasonal peak in transmission. Note that $t$ is indexed to $t=0$ on February 15, 2020.

To model pandemic fatigue, we set

$$
\begin{aligned}
& \kappa(t)=\bar{\kappa} *(1-\operatorname{normcdf}(t, \text { fatiguemean, fatiguesig }))+ \\
& \text { fatiguesize } * \bar{\kappa} * \text { normcdf }(t, \text { fatiguemean, fatiguesig })
\end{aligned}
$$


where $\bar{\kappa}$ sets the initial semi-elasticity of transmission with respect to daily deaths, fatiguesize sets the percentage reduction in this semi-elasticity in the long run, normcdf is the normal CDF, fatiguemean sets the date at which the transition in $\kappa(t)$ from its initial to new long run level is halfway complete, and fatiguesig sets the speed with which that transition occurs.

Initial conditions are $E(0)>0, E_{v}(0)=I(0)=I_{v}(0)=R(0)=H(0)=D(0)=0$, $S(0)=1-E(0)$. For the United States, $E(0)=33$ on February 15 out of a population of 330 million. For the United Kingdom, $E(0)=10$ on February 15 out of a population of 67.9 million.

I set the epidemiological parameters as follows: $\gamma=0.4, \sigma=0.425, \eta=0.025$, $\nu=0.2, \zeta=1 / 30$. The parameter $\sigma$ corresponds to an expected time before and exposed agent becomes infectious of 2.35 days and the parameter $\gamma$ corresponds to an expected time for which an infected individual is infectious of 2.5 days. These two parameters together imply a generation time of 4.85 days. These parameters are held constant for the US and the UK versions of the model. ${ }^{11}$

The parameter $\zeta$ corresponds to the rate at which those hospitalized flow either to death or recovery. This rate is chosen to have an average stay in compartment $H$ of 30 days, which corresponds to an average stay in the hospital of two weeks for those with serious illness and a reporting delay of deaths of two weeks. The infection fatality rate is given by $\eta \nu=0.005$ which is the product of a rate of serious illness of $2.5 \%$ of total infections and a fatality rate of $20 \%$ for those with serious illness. ${ }^{12}$ These parameters are also held constant for the US and the UK versions of the model.

The basic reproduction number of the virus at peak transmissibility is $\mathcal{R}_{0}(t)=\bar{\beta} / \gamma$

\footnotetext{
${ }^{11}$ See https://www.cdc.gov/coronavirus/2019-ncov/hcp/planning-scenarios.html. On that webpage, the CDC notes a mean time of approximately six days between symptom onset in one person to symptom onset in another person infected by that individual.

${ }^{12}$ See https://www.cdc.gov/coronavirus/2019-ncov/hcp/planning-scenarios.html. On that webpage, the CDC notes a median time from symptom onset to death of approximately two weeks and a median time from death to reporting just under three weeks.
} 
for the original virus and $\mathcal{R}_{0}(t)=\bar{\beta}_{v} / \gamma$ for the new variant. For the United States, I set $\bar{\beta}=3 \gamma$ giving a basic reproduction in Winter of 3. For the United Kingdom, I set $\bar{\beta}=3.6 \gamma$ giving a basic reproduction in Winter of 3.6. Both of these numbers are well within the range of estimates of this parameter from the early phase of the pandemic in both countries. For both countries, for the new variant of the virus, I set $\bar{\beta}_{v}=5 \gamma$ giving a basic reproduction in Winter of 5 . This implies that the new variant is $39 \%$ more transmissible than the original virus in the UK and $67 \%$ more transmissible than the original variant in the US. Note that the other epidemiological parameters associated with this new variant are assumed to stay the same, including the infection fatality rate.

To model seasonality of transmission in the United States, I set seasonalsize = 0.35 and seasonalposition $=20$. For the United Kingdom, I set seasonalsize $=1$ and seasonalposition $=15$. These parameters for seasonalposition set peak transmission rates in late January of each year.

The initial semi-elasticity of transmission with respect to daily deaths (measured as a fraction of the population) for the United States is $\bar{\kappa}=250000$. For the United Kingdom, this parameter is $\bar{\kappa}=140000$. To model the onset of pandemic fatigue in the United States, I set fatiguesize $=0.375$, fatiguemean $=285$ and fatiguesig $=$ 15. The corresponding parameters for the United Kingdom are fatiguesize $=0.575$, fatiguemean $=305$ and fatiguesig $=8$. 